\title{
ENTONACIÓN DE ENUNCIADOS INTERROGATIVOS EN EL ESPAÑOL DE MORELIA, MICHOACÁN ${ }^{1}$ INTERROGATIVE INTONATIONAL PATTERNS IN MORELIA, MICHOACÁN SPANISH
}

\author{
Érika Mendoza Vázquez \\ Universidad Nacional Autónoma de México \\ emvazquez03@gmail.com
}

Resumen

En este artículo se presenta una descripción de las configuraciones tonales de los enunciados interrogativos de la variedad de Morelia, Michoacán. La descripción contempla enunciados absolutos y pronominales con los subtipos: i) búsqueda de información (neutro), ii) confirmativo e iii) imperativo. Los datos de la prueba de completamiento discursivo muestran la realización del acento nuclear $\mathrm{L}+\mathrm{H}^{\star}$ en interrogativas absolutas neutras y de confirmación. Además se observa el alineamiento del tono de juntura final $\mathrm{H} \%$ para los tres tipos de enunciados interrogativos. Estos rasgos se distinguen del acento nuclear $\mathrm{L}^{\star}$ y el tono de juntura complejo $\mathrm{LH} \%$, documentados en interrogativas neutras e invitaciones en las variedades de Guadalajara (Orozco, 2017) y Ciudad de México (De la Mota et al., 2010).

Palabras Clave: interrogativas absolutas, interrogativas pronominales, dialectología, prosodia, variación, español de México

\section{Abstract}

This paper presents a description of the nuclear and prenuclear configurations of the interrogative intonation in Morelia, Michoacán Spanish. The description considers yes/no and wh- interrogatives with subtypes: i) information-seeking (neutral), ii) confirmatory and iii) imperatives. The data from discourse completion tasks show the realization of the nuclear accent $\mathrm{L}+\mathrm{H}^{\star}$ in yes-no questions neutral and confirmatory interrogatives. In addition, the alignment of the final boundary tone $\mathrm{H} \%$ is observed for the three types of interrogatives. These features are distinguished from the nuclear pitch accent $\mathrm{L}^{*}$ and the complex boun-

\footnotetext{
${ }^{1}$ Investigación realizada gracias al programa UNAM-DGAPA-PAPIIT IA400817, "Configuraciones tonales del español de México. Bases para una caracterización geoprosódica”. Agradezco los valiosos comentarios de dos dictaminadores anónimos, cuyas observaciones enriquecieron el contenido de este trabajo. Cualquier error $\mathrm{u}$ omisión en el texto es responsabilidad de la autora.
} 
dary tone LH\%, documented in yes-no questions neutral and invitations in the varieties of Guadalajara (Orozco, 2017) and Mexico City (De la Mota et al., 2010).

KEYwORDs: yes-no questions, wh- questions, dialectology, prosody, variation, Mexican Spanish

FECHA DE RECEPCIÓN: 2/08/2018

FECHA DE ACEPTACIÓN: 29/11/2018

\section{Introducción}

El objetivo de este trabajo es hacer una aproximación descriptiva de las configuraciones tonales de los enunciados interrogativos en la variedad del español de Morelia, Michoacán (México), ${ }^{2}$ obtenidos mediante la elicitación de una prueba de completamiento discursivo (Prieto y Roseano, 2009-2013; Martínez Celdrán y Fernández Planas, 2003-2018). Se presenta la caracterización entonativa de los enunciados interrogativos absolutos e interrogativos qu- (llamados parciales o pronominales), con los subtipos de preguntas: i) de búsqueda de información (neutra), ii) confirmativa y eco; e iii) imperativa - que incluyen las exhortativas- L La descripción basada en el modelo métrico autosegmental (Pierrehumbert, 1980; Ladd, 2008) y el sistema de notación prosódica Sp_ToBI (Beckman et al., 2002; Hualde y Prieto, 2015) permite establecer una comparación con los enunciados interrogativos de otras variedades de México -específicamente con datos de la Ciudad de México (De la Mota et al., 2010) y Guadalajara (Orozco, 2017)—.

\footnotetext{
${ }^{2}$ El estado de Michoacán se localiza en el occidente de México, tiene colindancia al norte con los estados de Colima, Jalisco, Guanajuato y Querétaro; al este con el Estado de México, con el Océano Pacífico al oeste; y con el estado de Guerrero, separado por el río Balsas, al sur. La ciudad de Morelia - cabecera municipal y capital del estado- se ubica en la región centro-norte de Michoacán; tiene una población de 784,776 habitantes (INEGI, 2015), de los cuales 3,811, mayores de 5 años son hablantes de una lengua indígena (purépecha, náhuatl y mazahua).
} 
En la clasificación entonológica tradicional del español, los enunciados interrogativos se han descrito considerando dos tipos básicos: i) el interrogativo absoluto y ii) el interrogativo $q u$ o pronominal (Navarro Tomás, 1944; Quilis, 1993). El primero se caracteriza por cinco puntos en la curva melódica: i) un inicio más alto en comparación con los enunciados aseverativos (Navarro Tomás, 1944; Quilis, 1993; Sosa, 1999; Face, 2004; Prieto, 2004), ${ }^{3}$ ii) el desplazamiento del pico tonal inicial hacia la sílaba postónica (Hualde y Prieto, 2015), iii) una declinación regular en la curva melódica desde el pico inicial hasta la última sílaba acentuada, iv) un nivel bajo en el F0 en la última sílaba tónica del enunciado; y v) el movimiento ascendente del F0 al final del enunciado. Acerca de la configuración global, Hualde y Prieto (2015) señalan que hay una tendencia a la desacentuación en el cuerpo del enunciado, que puede dar lugar a la reducción o eliminación de inflexiones tonales.

Por otro lado, los enunciados interrogativos qu- o pronominales del español se producen con una configuración final descendente - de forma similar al enunciado aseverativo. El primer pico tonal, el más prominente en el enunciado, coincide con la palabra interrogativa $q u$-, que concentra el foco de la pregunta (Sosa, 2003; NGLE, 2011). Respecto al movimiento de descenso en el cuerpo del enunciado, este abarca hasta la última sílaba tónica y puede realizarse en el rango mínimo del hablante (Sosa, 2003). Quilis (1993: 431) señala que el ascenso final en este tipo de interrogativas aporta un matiz de cortesía al enunciado.

Las configuraciones descritas anteriormente pueden mostrar variación motivada por factores dialectales y pragmáticos. ${ }^{4}$ En los últimos años, las descripciones entonativas

\footnotetext{
${ }^{3}$ Esta tendencia no ocurre de manera sistemática en todas las variedades del español. En la variedad de Canarias, se ha registrado que los enunciados interrogativos producidos en pruebas de lectura comienzan con un tono de juntura inicial bajo \%L (Dorta et al., 2018). En otras variedades, el inicio con una juntura inicial \%L -en pruebas de lectura- está motivado por factores sociales, tal es el caso del español de Cuba, donde las mujeres favorecen esta juntura inicial en interrogativas (Martín y Dorta, 2018).

${ }^{4}$ Respecto a la variación dialectal, se registran interrogativas absolutas con terminación descendente en el español de Puerto Rico, República Dominicana y Venezuela; y casos
} 
del español han considerado no únicamente la forma del enunciado, sino que también se integra una base pragmática que atiende al tipo de acto de habla o al grado de conocimiento que se presupone que el hablante tiene acerca de la respuesta. ${ }^{5}$ En el marco del proyecto ATLES (Prieto y Roseano, 2009-2013) se propone una clasificación de enunciados interrogativos neutros - búsqueda de información- y no neutros - preguntas de confirmación, órdenes e invitaciones- con distintos matices pragmáticos. Así se han registrado diferencias en los patrones entonativos de las preguntas de búsqueda de información y las preguntas de confirmación en distintas variedades del español. Por ejemplo, en el español de Puerto Rico (Armstrong, 2010) y Chile (Ortiz et al., 2010), los tonemas ! $\mathrm{H}^{\star} \mathrm{L} \%$ y $\mathrm{L}+\mathrm{H}^{\star} \mathrm{HH} \%$ registrados en preguntas de búsqueda de información, contrastan con el patrón descendente $\mathrm{H}+\mathrm{L}^{\star} \mathrm{L} \%$ de las preguntas de confirmación. Para el español de la Ciudad de México, la diferencia entre este tipo de preguntas se observa en el punto de ascenso de la juntura final; con la configuración $\mathrm{L}^{*} \mathrm{LH} \%$ para las interrogativas de búsqueda de información y con $\mathrm{L}^{*} \mathrm{H} \%$ en las de confirmación (De la Mota et al., 2010). Nótese que en ambos casos se mantiene la misma forma del acento nuclear con el tono bajo $\mathrm{L}^{*}$.

Otra propuesta de clasificación involucra el tipo de acto de habla y su influencia en la variación de las configuraciones tonales. Orozco describe los patrones entonativos de los enunciados interrogativos absolutos de las variedades de Monterrey, Guada-

de interrogativas $q u$ - con terminación ascendente en las variedades de República Dominicana, Chile y en la zona andina de Ecuador (Prieto y Roseano, 2009-2013). Por otro lado, desde las descripciones de Navarro Tomás (1944) se hacía referencia a la influencia del tipo pragmático de la interrogativa en la producción de las configuraciones tonales.

${ }^{5}$ Véanse trabajos inscritos en el marco de la metodología del Proyecto del Atlas Interactivo de la Entonación del Español (en adelante ATLES, Prieto y Roseano, 2009-2013). En México, el proyecto del Corpus Oral del Español de México (en adelante COEM, Martín Butragueño, Mendoza y Orozco, en preparación) adapta la base pragmática propuesta por ATLES para la elicitación de enunciados (Orozco, 2012). 
lajara y Tuxtla Gutiérrez, considerando tres tipos de actos ilocutivos - peticiones de información, de acción e invitaciones-. La autora reporta: i) las configuraciones $\mathrm{L}^{*} \mathrm{HH} \%$ y L* $\mathrm{LH} \%$ en las peticiones de información de Tuxtla y Guadalajara; en tanto que para las tres variedades se observa: ii) la recurrencia del patrón $\mathrm{L}^{*} \mathrm{H} \%$ en peticiones de acción y iii) el patrón $\mathrm{L}^{*} \mathrm{LH} \%$ en invitaciones (2017: 299).

Considerando el panorama expuesto anteriormente, en este trabajo se retoma la clasificación de Prieto y Roseano (20092013) con adaptaciones, siguiendo la propuesta de Escandell (1993) y la NGLE (2011). En este primer acercamiento a los datos se hace una descripción de base fonética y se expone una propuesta del repertorio de configuraciones tonales de los enunciados interrogativos absolutos y pronominales ( $q u-)$ en esta variedad del español. El uso del sistema de notación prosódica Sp_ ToBI tiene como fin establecer una comparación con datos de otras variedades de México. Con esta primera aproximación se busca contribuir con la descripción de las configuraciones tonales de una variedad del español que carece de estudios previos, y aportar información al ámbito de la geoprosodia del español de México.

\section{Antecedentes}

Configuraciones tonales de los enunciados interrogativos en el español de México

Los enunciados interrogativos absolutos neutros registrados en distintas variedades del español de México muestran características similares en la configuración global de la curva melódica. En los datos de la Ciudad de México (De la Mota et al., 2010) y Guadalajara (Orozco, 2017) se observa un tonema L* LH\%, es decir, una terminación ascendente precedida de un tono bajo, como se muestra en la figura 1. 


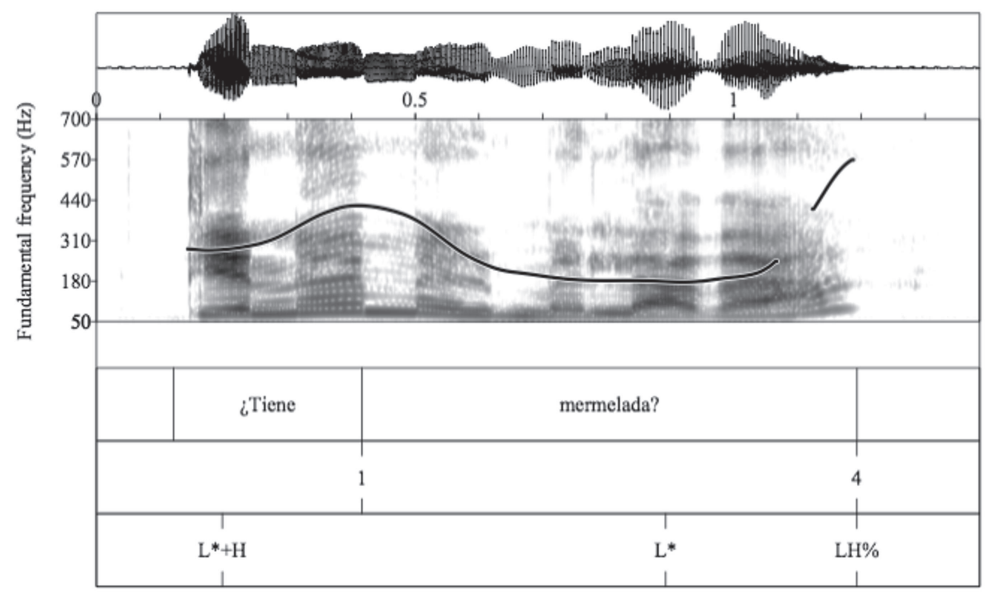

Figura 1. Oscilograma, espectrograma y curva melódica del enunciado ¿Tiene mermelada? Ciudad de México (Fuente: De la Mota et al., 2010: 330)

La curva melódica del enunciado interrogativo de la figura 1 muestra que en la palabra tiene, el primer pico tonal se realiza con un desplazamiento hacia la sílaba postónica $\left(\mathrm{L}^{*}+\mathrm{H}\right)$, seguido de un descenso que resulta en un tono bajo $\mathrm{L}^{*}$ en el acento nuclear. Obsérvese que el ascenso en la sílaba /da-/ comienza en la parte media de la vocal [a], y la primera parte de la sílaba se produce con un tono bajo, por lo tanto se considera un tono de juntura complejo LH\%.

En otras variedades de español de México se observan diferencias fonéticas en elementos locales del enunciado interrogativo neutro - en el primer acento tonal, acento nuclear o tono de juntura-. Así, en Cuapiaxtla, Tlaxcala (Mendoza, 2014), el descenso asociado con el acento nuclear $L^{*}$ de este tipo enunciativo, puede realizarse como un descenso más prominente dentro de la sílaba nuclear, con la forma $\mathrm{H}+\mathrm{L}^{*}$. En contraste, en datos de Monterrey se observa un tonema ascendente que comienza desde la sílaba nuclear del enunciado. Se registran dos realizaciones: i) con el acento nuclear alto $\mathrm{H}^{\star}$, seguido de un ascenso final $\mathrm{H} \%$ o sostenimiento del tono M\% (Orozco, 2017); y ii) con el acento nuclear $\mathrm{L}+\mathrm{H}^{\star} \mathrm{HH} \%$ con ascenso progresivo a lo largo de la sílaba nuclear, y una elevación mayor a 3 semitonos en la postónica (Montellano, 2017). 
Respecto a los enunciados interrogativos qu-neutros, en la variedad de la Ciudad de México se registra una configuración nuclear circunfleja $\mathrm{L}+\mathrm{H}^{\star} \mathrm{HL} \%$ (De la Mota et al., 2010). Esta realización se distingue de la configuración descendente $\mathrm{L}^{\star} \mathrm{L} \%$ (en términos del modelo métrico autosegmental y el sistema de notación Sp_ToBI), que se ha descrito en distintas variedades del español (cf. Sosa, 2003; Prieto y Roseano, 2009-2013; Hualde y Prieto, 2015). La figura 2 muestra un ejemplo de la configuración $\mathrm{L}+\mathrm{H}^{\star} \mathrm{HL} \%$.

El alineamiento del primer acento tonal del enunciado en la figura 2 se produce con el desplazamiento del pico tonal hacia la sílaba postónica $\left(\mathrm{L}^{\star}+\mathrm{H}\right)$; y a partir de su culminación, se observa una secuencia de acento tonales $\mathrm{H}^{\star}$ en el cuerpo del enunciado. En el tonema, el acento nuclear bitonal $\mathrm{L}+\mathrm{H}^{\star}$ se realiza con un ascenso progresivo a lo largo de la sílaba tónica, que se extiende hacia la postónica, seguido de un descenso dentro de la misma (HL\%).

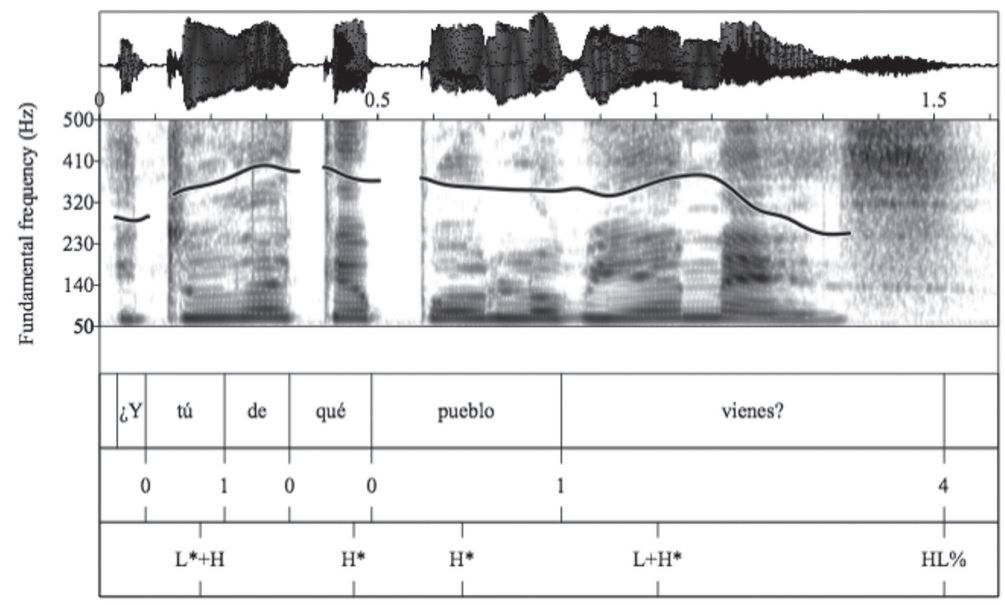

Figura 2. Oscilograma, espectrograma y curva melódica del enunciado ¿Y tú de qué pueblo vienes?, Ciudad de México (Fuente: De la Mota et al., 2010: 336)

Una tendencia que se ha registrado en distintas variedades del español de México es el inicio más alto de los enunciados interrogativos qu-, en comparación con los absolutos y los ase- 
verativos. ${ }^{6}$ Así, se ha propuesto el tono de juntura inicial \%H para los interrogativos qu-en variedades del centro de México en entornos urbanos - Puebla (Willis, 2008) - y en rurales - Tlaxcala (Mendoza, 2014) —. La frecuencia con la que se registra el tono $\% \mathrm{H}$ inicial en estas variedades, podría constituir una diferencia respecto a los interrogativos absolutos $-\mathrm{y}$ a otras variedades de México- en los cuales la tendencia es menos definida (cf. Ávila, 2003; Martín Butragueño, 2014a para la Ciudad de México).

\section{Metodología}

Acerca de la variedad de Morelia. Aspectos dialectales

En las distintas propuestas de zonas dialectales, la variedad de Michoacán se ha caracterizado como una zona aislada ${ }^{7}$ (cf. Lope Blanch, 1971) o como una división dialectal menor, junto con Jalisco (cf. Lope Blanch, 1996). En años recientes, Martín Butragueño (2014b) plantea - con datos fónicos - un modelo de análisis multivariable para la discusión de la zonificación dialectal del español de México, y ubica la variedad michoacana en la zona centro-oeste -integrada por Aguascalientes, Colima, Guanajuato, Guerrero, Hidalgo, Jalisco, Ciudad de México, Michoacán, Morelos y Querétaro-. Tomando en cuenta estas propuestas dialectales, en este trabajo se compararán las configuraciones tonales de la variedad de Morelia con dos variedades afines: Guadalajara, Jalisco — siguiendo la clasificación de Lope Blanch (1996) - y Ciudad de México — de acuerdo con la propuesta de Martín Butragueño (2014b)—. Con la presente descripción se busca hacer una contribución en el ámbito de la

\footnotetext{
${ }^{6}$ No es un hecho sistemático, pues en datos de la Ciudad de México, se ha reportado que en estilo de lectura, los enunciados aseverativos pueden tener inicios más altos que los interrogativos (Martín Butragueño, 2014a).

${ }^{7}$ Sin embargo, el autor cuestiona la autonomía de esta variedad como zona y sugiere que podría considerarse como una variante de las hablas occidentales - que correspondería con los estados de Jalisco, Colima y Nayarit (Lope Blanch, 1979)—.
} 
entonación en esta zona dialectal, ${ }^{8}$ identificando aspectos semejantes y divergentes, que en futuras investigaciones puedan apoyar una propuesta geoprosódica del español de México.

\section{Informantes e instrumentos de elicitación}

La muestra de datos que se utiliza para este trabajo es parte del Corpus Oral del Español de México (COEM, Martín Butragueño, Mendoza y Orozco, en preparación). Los datos provienen de seis informantes originarios de Morelia — tres hombres y tres mujeres - con una estratificación por tres grupos de edad: i) jóvenes (20-34 años), ii) adultos (35 a 54 años) y iii) mayores (55 años o más). Se considera una muestra de 120 enunciados interrogativos, a razón de 20 por informante. ${ }^{9}$ Además se incluyen 60 enunciados aseverativos (10 por hablante) para el cálculo del tono de juntura inicial.

Para la elicitación de datos se llevó a cabo una prueba de encuesta de situaciones - o cuestionario inductivo (Prieto y Roseano, 2009-2013; Martínez Celdrán y Fernández Planas, 2003-2018) - Este instrumento consiste en una tarea de completamiento discursivo, en la que el entrevistador le presenta al informante una serie de contextos, con el fin de obtener distintos tipos enunciativos y matices pragmáticos. Un ejemplo de las situaciones se muestran en (1) y (2):

(1) Entras a una tienda y le preguntas a un empleado si tiene mermelada (Respuesta esperada: ¿Tiene mermelada? / Disculpe, ¿tiene mermelada?) Interrogativa absoluta neutra.

(2) Un amigo tuyo acaba de rentar su departamento. Pregúntale a quién se lo rentó (Respuesta esperada: ¿A quién se lo rentaste?) Interrogativa $q u$ - neutra.

\footnotetext{
${ }^{8}$ Para la descripción de otros patrones entonativos de la variedad de Michoacán, véase el trabajo de Coronado (2014) acerca de los enunciados aseverativos en Pátzcuaro, isla de Janitzio y Santa Fe de la Laguna.

${ }^{9}$ Se seleccionaron 10 enunciados interrogativos absolutos - dos neutros, cuatro de confirmación y cuatro imperativas- y 10 interrogativos qu-, que incluyen seis interrogativas neutras, una de confirmación y tres imperativas.
} 
Con base en la clasificación de Prieto y Roseano (20092013), los enunciados interrogativos absolutos e interrogativos $q u$ - obtenidos en la elicitación pueden caracterizarse como: i) neutros (búsqueda de información), ii) no neutros (preguntas de confirmación) y iii) preguntas imperativas (órdenes e invitaciones). Los dos primeros implican la búsqueda de información, pero hay una diferencia en el grado de conocimiento acerca de la respuesta (Escandell, 1993). Así, en las interrogativas neutras se asume un menor conocimiento real - por parte del emisory mayor suposición sobre el conocimiento - por parte del destinatario (Prieto y Rigau, 2011) - . En contraste, las preguntas confirmativas involucran un mayor grado de certeza por parte del hablante y se busca que el interlocutor confirme la información (Escandell, 1993). Para este trabajo se incluyen las interrogativas eco dentro de las interrogativas de confirmación, pues se considera que, al repetir el contenido del enunciado previo, el hablante tiene una idea acerca de la respuesta. Si bien por medio de la formulación de la interrogativa eco se puede confirmar una información que el hablante supone, también es posible que se exprese una intención secundaria - sorpresa, incredulidad, indignación o desconfianza - ante lo que ha enunciado el interlocutor previamente.

Por otro lado, en las preguntas imperativas, el objetivo pragmático no es la búsqueda de información, sino lograr que el interlocutor realice una acción (petición de acción, en términos de Orozco, 2017). Así, se distinguen las órdenes, con una mayor imposición por parte del hablante, frente a las invitaciones, donde la acción propuesta puede beneficiar al interlocutor. ${ }^{10}$

Aunque se reconoce la importancia de trabajar con datos obtenidos en contextos naturales o espontáneos (Martín Butragueño, 2014b), también se considera que en un primer acercamiento a una variedad que no cuenta con descripciones previas, es pertinente contar con una fuente de datos que permita comparar un mismo tipo de enunciado (o con un matiz pragmático

\footnotetext{
${ }^{10}$ Esta distinción se retoma de la clasificación de Prieto y Roseano (2009-2013), donde las preguntas imperativas contemplan los subtipos de órdenes y peticiones.
} 
similar) entre diferentes hablantes. Una ventaja del uso del cuestionario inductivo, para este trabajo, es que es posible comparar los datos con las descripciones de las variedades del español de la Ciudad de México (De la Mota et al., 2010) y Guadalajara (Orozco, 2017), que se basan en la misma metodología de elicitación.

\section{Transcripción de datos y análisis instrumental}

El análisis instrumental se llevó a cabo con el programa Praat, versión 6.0.16 (Boersma y Weenink, 2016), y para la transcripción de datos se utiliza el sistema de notación prosódica Sp_ToBI (Beckman et al., 2002; Hualde y Prieto, 2015), que está basado en los postulados del modelo métrico-autosegmental (Pierrehumbert, 1980; Ladd, 2008). Aunque dicho modelo y notación prosódica tienen una base fonológica, para este trabajo se considera su alcance descriptivo en un nivel fonético, en un estado previo a la fonologización de los datos. En este sentido, se asume que la diferencia en la realización de una configuración tonal no implica necesariamente un contraste fonológico; sino que puede atribuirse a diferencias motivadas en el nivel fonético, donde se observarán tanto las particularidades de los datos de Morelia, como las semejanzas y diferencias con otras variedades del español de México.

La transcripción incluye tres niveles: i) ortográfico - por sílabas-, ii) separación prosódica ${ }^{11}$ y iii) nivel tonal - acentos tonales y tonos de juntura- (véanse figuras 1 y 2 supra). La base fonética de la descripción incorpora la medición (en hercios, hz) de distintos puntos en el material prenuclear y nuclear: i) la frecuencia fundamental (F0) del inicio absoluto del enunciado

\footnotetext{
${ }^{11}$ Para este nivel se utiliza la jerarquía prosódica propuesta por Nespor y Vogel (1994) y Gussenhoven (2004), así como las convenciones del sistema Sp_ToBI (Hualde y Prieto, 2015). Se utilizan los índices en una escala de 0 a 4 para dar cuenta de: ausencia de lindes (nivel 0), separación entre palabras fonológicas (1), frases entonativas menores (3) y frases entonativas mayores, o final de enunciado (4). El índice 2 se utiliza en el nivel fonético para representar lindes que pueden corresponder con la frase entonativa menor, pero en la realización no se observa una pausa que pueda justificar la marcación con el índice 3.
} 
(sílaba tónica o átona) y ii) el F0 del inicio, centro y final de cada sílaba tónica y la postónica en el inicio, cuerpo y tonema del enunciado. Con base en estas medidas, se toma como referencia el primer pico tonal y el tonema para calcular la diferencia en semitonos (st) entre: i) el inicio, centro y final de cada sílaba tónica, y ii) el centro/final de la tónica respecto al centro/final de la postónica. ${ }^{12}$ Además, se considera el umbral de percepción de 1.5 st (Pamies et al., 2001; Murrieta, 2016) para establecer la diferencia entre acentos monotonales y bitonales. En el nivel de descripción fonética, las diferencias mayores a 3 st en acentos tonales (cf. Martín Butragueño, 2011), y de 5 st en tonos de juntura se marcan con los diacríticos de upstep (i) o downstep (!), como se explicará en los siguientes apartados.

\section{Resultados}

Repertorio de acentos tonales y tonos de juntura

El repertorio de acentos tonales y tonos de juntura en los enunciados interrogativos de la variedad de Morelia está basado en la propuesta de Hualde y Prieto (2015). En el cuadro 1 se presentan los acentos monotonales registrados en la muestra de datos. ${ }^{13} \mathrm{Se}$ observa la realización del acento nuclear $\mathrm{L}^{*}$, que también se ha documentado en los enunciados interrogativos neutros en las variedades del español mexicano central —en la Ciudad de México (De la Mota et al., 2010), Puebla (Willis, 2008) y Cuapiaxtla, Tlaxcala (Mendoza, 2014) - . Orozco (2017) registra este acento nuclear $\mathrm{L}^{\star}$ en peticiones de información, acción e invitaciones en datos de las variedades de Guadalajara y Tuxtla Gutiérrez.

\footnotetext{
${ }^{12}$ El cálculo en semitonos permite estandarizar los valores en hercios, con el fin de comparar un mismo tipo de movimiento, independientemente del nivel de la frecuencia fundamental con el que se produce. Así es posible neutralizar diferencias en el rango de voz de hombres y mujeres.

${ }^{13}$ Las representaciones esquemáticas se basan en la propuesta de Estebas Vilaplana y Prieto (2008). La casilla sombreada indica la sílaba tónica en acentos tonales, y la postónica en el tono de juntura.
} 


\begin{tabular}{|c|c|c|}
\hline Esquema & Transcripción & Descripción \\
\hline & $\mathrm{L}^{*}$ & $\begin{array}{l}\text { Este acento se caracteriza por un } \\
\text { valor bajo del F0 derivado de un } \\
\text { descenso progresivo o del sosteni- } \\
\text { miento de un valor bajo de F0 de } \\
\text { la sílaba pretónica. Se produce en } \\
\text { el rango mínimo del hablante. Se } \\
\text { registra en posición nuclear. }\end{array}$ \\
\hline & $\mathrm{H}^{*}, ! \mathrm{H}^{*}$ & $\begin{array}{l}\text { El acento tonal } \mathrm{H}^{*} \text { se caracteriza } \\
\text { por la producción del F0 en un } \\
\text { rango elevado. También se realiza } \\
\text { con la forma } ! \mathrm{H}^{*} \text {; es decir, con un } \\
\text { ascenso menor a } \mathrm{H}^{*} \text {. Se registra en } \\
\text { posición nuclear y prenuclear. }\end{array}$ \\
\hline
\end{tabular}

Cuadro 1. Acentos monotonales

Por otro lado, se registra el acento monotonal $\mathrm{H}^{\star}$, con la variante $! \mathrm{H}^{\star}$, en posición nuclear y prenuclear. Llama la atención que como primer acento tonal se registra con mayor recurrencia en los datos de jóvenes y adultos - tanto en hombres como en mujeres - principalmente en enunciados interrogativos $q u-$. En tanto que el número de acentos tonales $\mathrm{H}^{\star}$ disminuye en datos de personas mayores - cinco casos en datos de mujer y sólo uno en datos de hombre-. Para la variedad de Guadalajara, Orozco (2017: 91) reporta la realización de este acento $\mathrm{H}^{*}$ en posición prenuclear en peticiones de acción.

En posición nuclear, para las interrogativas absolutas se observó el acento tonal $\mathrm{H}^{\star}\left(\mathrm{o} ! \mathrm{H}^{\star}\right)$ en órdenes e invitaciones (véanse figuras 7 y 8 infra) en los datos de cinco hablantes, excepto en los enunciados del hombre mayor, que registró el acento nuclear $\mathrm{L}+{ }_{j} \mathrm{H}^{*}$. La realización del acento nuclear $\mathrm{H}^{*}$ también se reporta en Guadalajara para invitaciones (Orozco, 2017: 296). En este sentido, para las invitaciones, los acentos nucleares de las variedades de Guadalajara y Morelia difieren de la Ciudad de México, en la que se documenta el tonema $\mathrm{L}^{*}{ }_{\mathrm{i}} \mathrm{H} \%$, con acento nuclear bajo (De la Mota et al., 2010). ${ }^{14}$

${ }^{14}$ En la descripción de la Ciudad de México, se emplea la notación HH\% para dar cuenta de un ascenso prominente, en el nivel más alto del rango del hablante (De la 
Por otro lado, el repertorio de acentos bitonales incluye realizaciones con desplazamiento hacia la sílaba postónica, $\mathrm{L}+<\mathrm{H}^{\star}$ y $\mathrm{L}^{\star}+\mathrm{H}$, ambos en posición prenuclear (véase cuadro 2 ). Estos acentos bitonales también se han documentado en la variedad de la ciudad de México (De la Mota et al., 2010) para enunciados interrogativos en general, y en datos de Guadalajara para peticiones de información e invitaciones - con forma de interrogativa absoluta - (Orozco, 2017). Para la variedad de Morelia, se registra el acento bitonal con alineamiento dentro de la sílaba tónica $\mathrm{L}+\mathrm{H}^{\star}$, con la variante $\mathrm{L}+{ }_{j} \mathrm{H}^{\star}$-en casos de ascensos superiores a 3 semitonos-. Cabe mencionar que en posición prenuclear, la realización del acento bitonal $\mathrm{L}+\left(\mathrm{j}_{\mathrm{j}}\right) \mathrm{H}^{\star}$ se registró en 38 enunciados -en 23 casos de interrogativas absolutas (de confirmación e imperativas) y 15 de interrogativas $q u$ - (neutras e imperativas)—. En términos cualitativos, se observa que en las preguntas absolutas de confirmación y en las interrogativas $q u$ - neutras, el alineamiento al interior de la sílaba tónica del acento tonal $\mathrm{L}+(\mathrm{i}) \mathrm{H}^{*}$ y su ascenso prominente se asocia con una mayor expresividad del hablante (cf. NGLE, 2011: 469; Martín Butragueño, 2016).

\begin{tabular}{|c|c|c|}
\hline Esquema & Transcripción & Descripción \\
\hline & $\mathrm{L}+<\mathrm{H}^{*}$ & $\begin{array}{l}\text { Este acento tonal se caracteriza } \\
\text { por un ascenso progresivo del } \\
\text { F0 a lo largo de la sílaba tóni- } \\
\text { ca, que se desplaza y culmina } \\
\text { en la postónica (delayed peak). } \\
\text { Se registra en posición prenu- } \\
\text { clear. }\end{array}$ \\
\hline
\end{tabular}

Mota et al., 2010: 321). En este trabajo se hace la adaptación de la notación —con el diacrítico de upstep - según la propuesta de Hualde y Prieto (2015). 


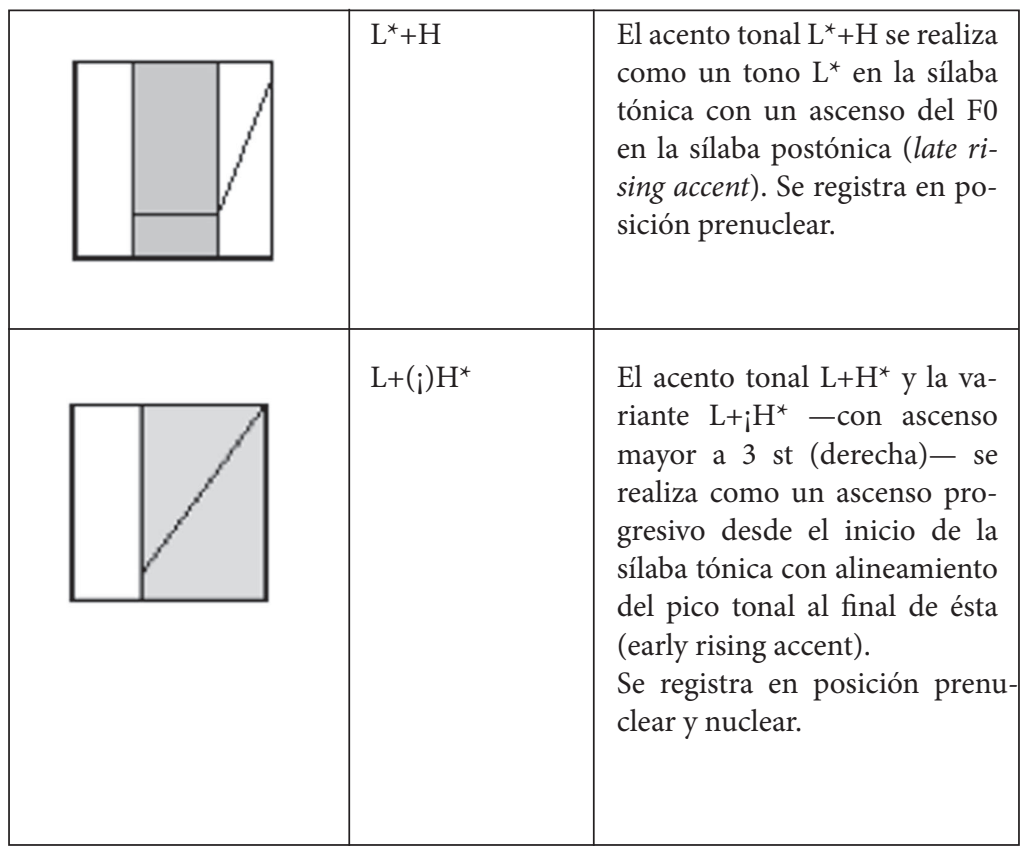

Cuadro 2. Acentos bitonales

En posición nuclear, se registra el acento bitonal $\mathrm{L}+\left({ }_{\mathrm{j}}\right) \mathrm{H}^{*}$ en 50 casos - donde 20 enunciados corresponden a interrogativas $q u-$ y 30 a interrogativas absolutas-. Esta realización es una diferencia respecto a la variedad de la ciudad de Guadalajara, donde no se documentan casos con el acento nuclear bitonal $\mathrm{L}+\mathrm{H}^{*}$ en interrogativas absolutas (cf. Orozco, 2017). Para la Ciudad de México, el acento nuclear bitonal $\mathrm{L}+\mathrm{H}^{*}$ se reporta en interrogativas qu- neutras, imperativas e invitaciones (cf. De la Mota et al., 2010). Con base en la muestra de datos, se puede sugerir que la realización del acento nuclear bitonal $\mathrm{L}+\left(_{\mathrm{i}}\right) \mathrm{H}^{*}$ en enunciados interrogativos absolutos establece una diferencia - en principio de carácter fonético- en la variedad de Morelia frente a las de Guadalajara y Ciudad de México (compárense las figuras 1 y 4$)$.

En relación con los tonos de juntura, se observa que el repertorio incluye únicamente formas monotonales (véase cuadro 3). Esta realización constituye una diferencia fonética, frente al 
tono de juntura complejo LH\% documentado en las preguntas absolutas neutras, preguntas eco e imperativas, en datos de la Ciudad de México (De la Mota et al., 2010), y en las peticiones de información e invitaciones en la variedad de Guadalajara (Orozco, 2017).

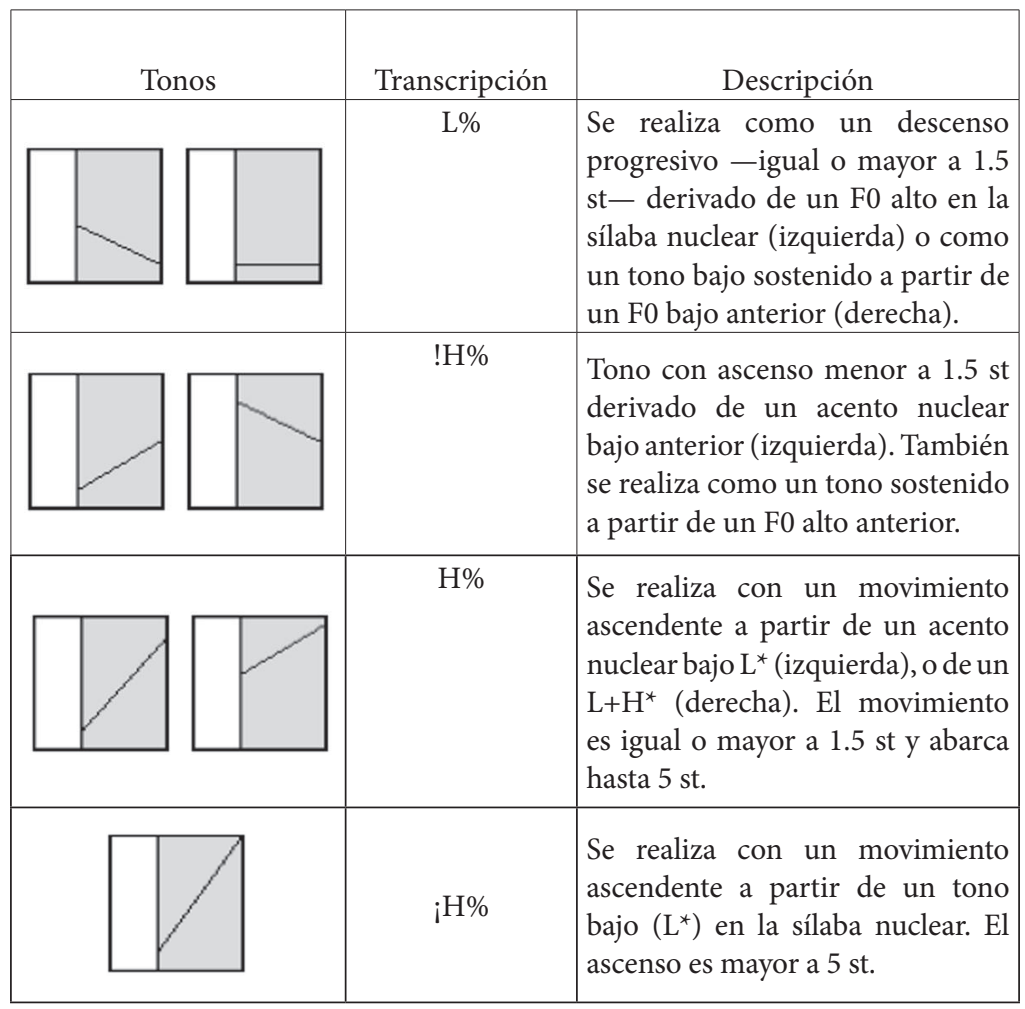

Cuadro 3. Tonos de juntura

Como se mencionó en el apartado de metodología, la asignación de acentos tonales y tonos de juntura se apoya en el parámetro fonético de 1.5 st para establecer una diferencia tonal (Pamies et al., 2001; Murrieta, 2016). Así, se han establecido como umbrales: i) para el tono de juntura bajo L\%, el descenso igual o mayor a $1.5 \mathrm{st}$, que se distingue de ii) el tono de juntura ! $\mathrm{H} \%$, asignado cuando el movimiento de ascenso es menor a 1.5 st o como el sostenimiento del acento nuclear alto $\mathrm{H}^{\star}$ 
o ! $\mathrm{H}^{\star}$ anterior. Por otro lado, el tono de juntura $\mathrm{H} \%$ se marca considerando un rango en el movimiento ascendente de 1.5 a 5 st. En los casos donde el movimiento supera el rango de $5 \mathrm{st}$, entonces se marca el tono de juntura ${ }_{¡} \mathrm{H} \%$. Estos dos últimos umbrales se determinaron con base en el valor del ascenso en la interrogativa absoluta neutra, con un movimiento de máximo 5 st, que contrasta con el rango de ascenso de las interrogativas no neutras - que pueden alcanzar hasta 9 st en preguntas $q u$ - de confirmación.

El repertorio de acentos tonales y tonos de juntura que se ha mostrado en los cuadros 1 a 3 da cuenta de elementos locales que distinguen a los enunciados interrogativos de la variedad de Morelia. La integración de este repertorio en la configuración global del enunciado se presenta en los siguientes apartados de descripción.

\section{Tono de juntura inicial en enunciados interrogativos de Morelia}

En líneas anteriores se mencionó que en distintas variedades del español de México se ha observado que los enunciados interrogativos comienzan en un nivel más alto que los aseverativos (Willis, 2008; Mendoza, 2014; Montellano, 2017). Con el fin de comprobar si este rasgo se distingue en la variedad de Morelia, se midieron los inicios de una muestra de 180 enunciados (30 enunciados x 6 informantes), a razón de 60 interrogativos absolutos, 60 interrogativos $q u$ - y 60 aseverativos neutros. Estos últimos se incluyen para calcular el tono normal ${ }^{15}$ o rango medio del hablante (Sosa, 1999; Ávila, 2003; Orozco, 2008).

Siguiendo la metodología propuesta por Ávila (2003) y Orozco (2008), se toma como parámetro el promedio del tono normal de cada hablante para asignar el tono de juntura inicial. Considerando el inicio del enunciado, en los casos donde el va-

\footnotetext{
${ }^{15}$ El tono normal o rango medio del hablante se refiere al promedio de la altura tonal con la que se comienza un enunciado neutro (cf. Zamora y Guitart, 1982). Una metodología para el cálculo de este tono normal es obtener el promedio de diez enunciados aseverativos neutros - cinco con sílaba tónica y cinco con sílaba átona, en posición de inicio absoluto (Ávila, 2003).
} 
lor del F0 (en hz) es mayor o igual al promedio del tono normal, se asigna un tono $\% \mathrm{H}$. Si el valor es menor al promedio, se asigna un tono bajo \%L. ${ }^{16}$ Los resultados para cada tipo enunciativo se muestran en el cuadro 4.

\begin{tabular}{cccc}
\hline $\begin{array}{c}\text { Tono de Juntura } \\
\text { inicial }\end{array}$ & $\begin{array}{c}\text { Interrogativos } \\
\text { absolutos }\end{array}$ & $\begin{array}{c}\text { Interrogativos } \\
q u-\end{array}$ & Aseverativos \\
\hline $\mathbf{\% L}$ & $18(30 \%)$ & $4(6.7 \%)$ & $38(63.3 \%)$ \\
$\mathbf{\% H}$ & $42(70 \%)$ & $56(93.3 \%)$ & $22(36.7 \%)$ \\
Total & $60(100 \%)$ & $60(100 \%)$ & $60(100 \%)$ \\
\hline
\end{tabular}

Chi cuadrado $=43.8, p=<0.00001$

Cuadro 4. Tono de juntura inicial en los tres tipos enunciativos $(\mathrm{N}=180)$

Los resultados del cuadro 4 indican una tendencia a la realización de un inicio más alto en los enunciados interrogativos, que se marca con una juntura inicial $\% \mathrm{H}$, y la significatividad de la relación entre la variable tono de juntura y tipo enunciativo ( $p$ $=<0.00001)$. El mayor número de casos de juntura inicial $\% \mathrm{H}$ se registra en los enunciados interrogativos $q u$-, seguido del interrogativo absoluto y en menor medida el enunciado aseverativo. Esta tendencia de inicio más alto en interrogativos qu- sigue lo que se ha documentado para otras variedades del español de México en hablas centrales (Willis, 2008; Mendoza, 2014).

$\mathrm{La}$ distribución de tonos de juntura inicial \% $\mathrm{H}$ y $\% \mathrm{~L}$ en los tres tipos enunciativos muestra que no es un hecho categórico que las interrogativas se realicen con un inicio más alto que las aseverativas, pues también se observan casos de $\% \mathrm{H}$ en estas. Este hecho es similar al que señala Martín Butragueño (2014a) para la Ciudad de México, donde, en datos de lectura, se reportan casos de aseverativas con inicios $\% \mathrm{H}$. En la variedad de Morelia, los datos expuestos anteriormente apoyan la tendencia

\footnotetext{
${ }^{16}$ Para este trabajo no se determinó un umbral de referencia en las diferencias entre los promedios, sólo se consideró el nivel en hz. A partir de los comentarios de los dictaminadores se hizo la comparación entre los promedios, y con la fórmula de Nooteboom (1997), se observó una diferencia de 1 a 1.5 st. entre el tono normal y el inicio de las interrogativas.
} 
que apunta a inicios más altos en interrogativos $q u$-, seguidos de interrogativos absolutos. En las transcripciones que se presentan en los siguientes apartados, se marcará la juntura inicial $\% \mathrm{H}$ en un nivel fonético, atendiendo al tono normal de cada hablante.

\section{Configuraciones tonales de enunciados interrogativos absolutos}

\section{Interrogativa neutra (de búsqueda de información)}

En la interrogativa absoluta neutra se registran dos configuraciones nucleares, en ambos casos con un tono de juntura alto $\mathrm{H} \%$. La realización más frecuente es el tonema $\mathrm{L}+\mathrm{H}^{*} \mathrm{H} \%-$ con un movimiento de 2.5 semitonos en promedio en el acento nuclear bitonal—, seguido de un ascenso en el tono de juntura - con un promedio de $3.5 \mathrm{st}$, pero que puede alcanzar hasta $5 \mathrm{st}-$. La segunda realización ocurre con el acento nuclear monotonal $\mathrm{L}^{*}$ $\mathrm{H} \%$ y se registró en los datos del hombre joven y del adulto. ${ }^{17}$ Esta configuración tiene similitud con el acento nuclear del tonema $\mathrm{L}^{*} \mathrm{LH} \%$ registrado en la variedad de la Ciudad de México (De la Mota et al., 2010) y Guadalajara (Orozco, 2017). Por otro lado, en el material prenuclear se registra el desplazamiento del pico tonal $\mathrm{L}+<\mathrm{H}^{*} \mathrm{o} \mathrm{L}^{*}+\mathrm{H}$, de manera similar a los enunciados interrogativos de la Ciudad de México.

En términos fonéticos, las configuraciones documentadas en la variedad de Morelia muestran una diferencia respecto a la variedad del centro y la de Guadalajara en el punto de ascenso en el tono de juntura final. En la variedad de Morelia, el ascenso final de $\mathrm{H} \%$ ocurre al inicio de la sílaba postónica, como se observa en el enunciado de la figura 3 (compárese con el enunciado de la figura 1 supra de la Ciudad de México).

\footnotetext{
${ }^{17}$ Para este tipo enunciativo sólo se registró un caso con acento nuclear monotonal $\mathrm{H}^{*}$.
} 


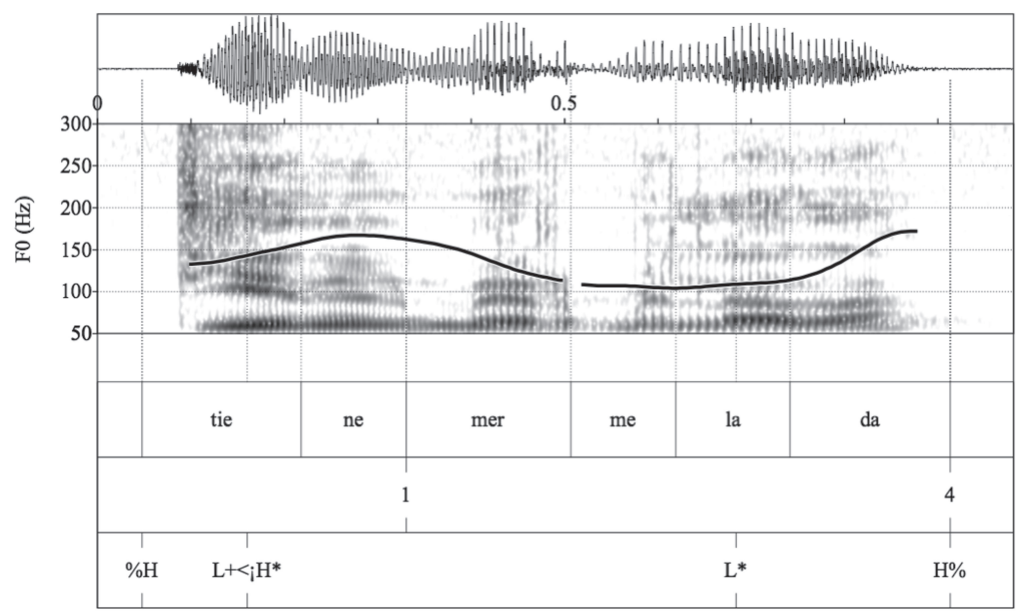

Figura 3. Oscilograma, espectrograma y curva melódica del enunciado ¿Tiene mermelada?, producido por un hombre, adulto ${ }^{18}$

En el material prenuclear ${ }^{19}$ del enunciado de la figura 3 se observa que el primer acento tonal se realiza con un desplazamiento del pico tonal, $\mathrm{L}+<_{i} \mathrm{H}^{*}$, con un ascenso de 3.6 st. El tonema tiene un acento nuclear bajo $\mathrm{L}^{\star}$, seguido del tono de juntura $\mathrm{H} \%$, con un ascenso progresivo - de 4.8 semitonos- que comienza al inicio de la sílaba /-da/. Esta realización con el ascenso al inicio de la sílaba postónica en el tonema es diferente de la juntura LH\% registrada en la variedad de la Ciudad de México para este tipo enunciativo (De la Mota et al., 2010), cuyo ascenso ocurre en la parte media de la sílaba postónica.

$\mathrm{El}$ acento nuclear $\mathrm{L}+\mathrm{H}^{*}$ es el más recurrente para el enunciado interrogativo absoluto neutro en esta variedad del español. En la realización de este acento nuclear se observa que el ascenso es moderado, no mayor a 3 st, en contraste con las inte-

\footnotetext{
${ }^{18}$ La escala de la ventana de transcripción se ajusta de acuerdo con el rango de voz del hablante. Para datos de hombre se utiliza la escala de 50 a $300 \mathrm{hz}$, y para mujer 75 a 500 hz. En casos de mayor expresividad se amplía la escala. Las figuras se elaboraron con el script CreaFigures Script.praat [Praat script] (Welby, 2003).

${ }^{19}$ Para este enunciado, la marcación del tono de juntura inicial \% $\mathrm{H}$ se apoya en la diferencia de 1.6 st entre el promedio del tono normal del hablante $(123 \mathrm{hz})$ y el inicio de la interrogativa $(136 \mathrm{hz})$.
} 
rrogativas no neutras (véase figura 5 infra). El enunciado de la figura 4 muestra el tonema $\mathrm{L}+\mathrm{H}^{*} \mathrm{H} \%$, con un ascenso de $1.5 \mathrm{st}$ en el acento nuclear.

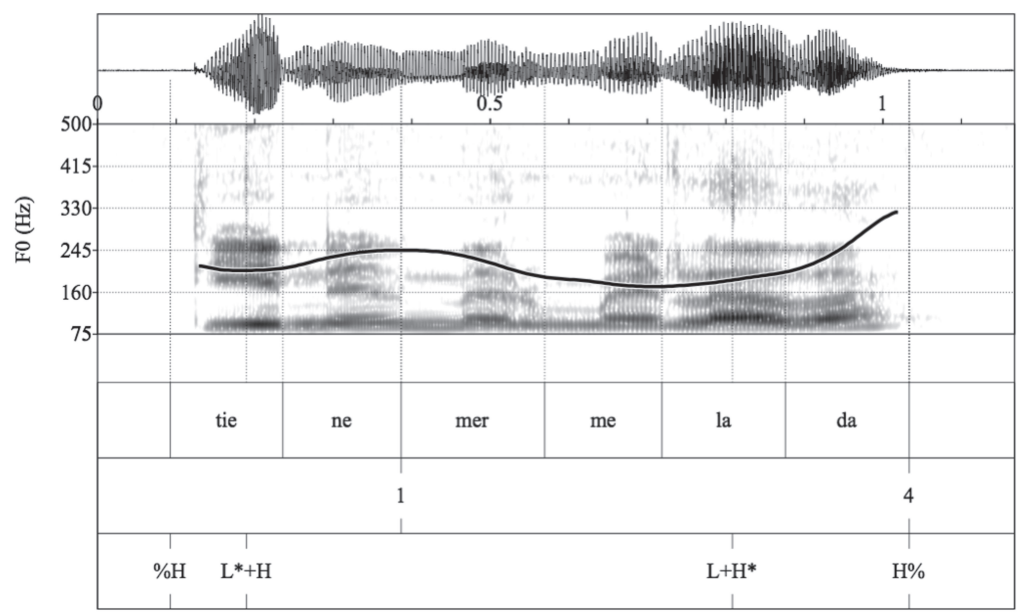

Figura 4. Oscilograma, espectrograma y curva melódica del enunciado ¿Tiene mermelada?, producido por una mujer, adulta

En el enunciado interrogativo de la figura 4 podemos apreciar una configuración global similar al de la figura 3. En ambos casos se produce un desplazamiento del primer pico tonal y un ascenso prominente en la juntura final — de 4.6 st en la figura 4-, que comienza en la parte inicial de la sílaba postónica. La diferencia se observa en elementos locales, específicamente en la forma del primer acento tonal $\mathrm{L}^{*}+\mathrm{H}$, con un tono bajo en la sílaba tónica /tie-/ y la culminación del pico tonal en la postónica. La segunda diferencia se percibe en el acento nuclear, bitonal en este enunciado; en contraste con el acento nuclear bajo $\mathrm{L}^{*}$ documentado en la variedad de la Ciudad de México y Guadalajara. En cuanto al tono de juntura, el promedio de ascenso de 3.5 st es similar al reportado en la variedad de Guadalajara (Orozco, 2017: 290), pero en esta última, el movimiento puede alcanzar hasta 10 st —el doble del nivel máximo que se registra en Morelia. 
Si consideramos que en la muestra de datos de Morelia, el tono de juntura final $\mathrm{H} \%$ se realiza con un tipo de adelantamiento del ascenso - comparado con el tono LH\% de la ciudad de México y Guadalajara-, surge una pregunta acerca de si la realización del acento nuclear $\mathrm{L}+\mathrm{H}^{\star}$ puede analizarse como una forma aún más adelantada del tono de juntura. Esta propuesta de ascenso preparatorio se apoyaría por el ascenso moderado del acento nuclear $\mathrm{L}+\mathrm{H}^{*}$, y por la forma de la curva melódica. En una investigación posterior se podría comprobar con una prueba de percepción si el ascenso preparatorio se asocia con un tipo enunciativo o con un rasgo de indización.

\section{Interrogativa absoluta de confirmación}

En las preguntas de confirmación se registran tres tonemas: i) $\mathrm{L}+(\mathrm{i}) \mathrm{H}^{*} \mathrm{H} \%$, ii) $\mathrm{H}^{*} \mathrm{H} \%$ y iii) $\mathrm{L}^{*} \mathrm{H} \%$. En estas configuraciones, el tono de juntura $\mathrm{H} \%$ no presenta ascensos prominentes, sino que se realiza con un promedio de 2.8 semitonos. En función de características individuales de los hablantes, se observan particularidades en este tipo de interrogativa. El promedio de ascenso del acento nuclear bitonal $\mathrm{L}+\mathrm{H}^{\star}$ es de 2 semitonos para esta interrogativa; sin embargo, en los datos de la mujer mayor, el enunciado se percibe con una mayor fuerza expresiva, y se produce el acento nuclear bitonal $\mathrm{L}+{ }_{i} \mathrm{H}^{*}-$ con un ascenso de 5 st - seguido de la juntura final $\mathrm{H} \%$ - con ascenso de 3 st (véase figura 5) - . Un hecho que llama la atención es que a pesar de que en los datos de la mujer mayor se presentan las inflexiones más prominentes en el enunciado, no se observa la juntura inicial \%H. Por otro lado, en los enunciados de los hablantes de los otros grupos de edad, y del hombre mayor, no se registran ascensos prominentes, pero sí se registra el tono \%H inicial. En estos casos se percibe una expresividad más moderada. 


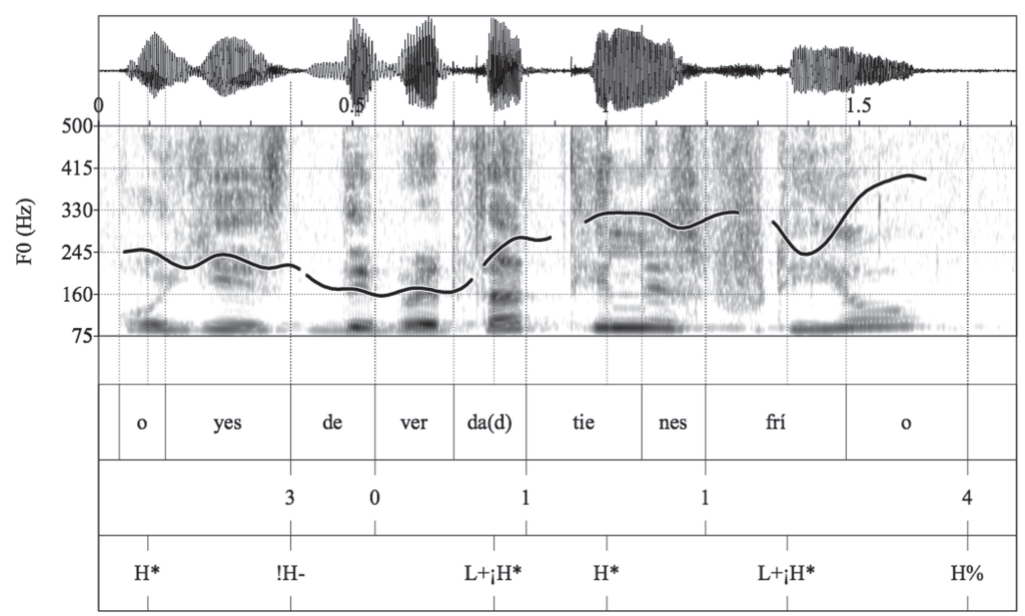

Figura 5. Oscilograma, espectrograma y curva melódica del enunciado Oyes, ¿de verdad tienes frío?, producido por una mujer, mayor

En el pretonema del enunciado de la figura 5 se observa una secuencia de acentos tonales alineados en la sílaba tónica, el bitonal $\mathrm{L}+\mathrm{H}^{*}$ y el monotonal $\mathrm{H}^{*}$, diferentes a los acentos tonales con pico desplazado $\mathrm{L}+<\mathrm{H}^{\star}$, registrados en la interrogativa neutra. Este alineamiento en los acentos prenucleares también se ha registrado para las interrogativas absolutas de confirmación de la variedad de la Ciudad de México (De la Mota et al., 2010). En ese sentido, el alineamiento al interior de la sílaba tónica y el valor del movimiento puede ser un recurso para enfatizar la expresividad del enunciado (cf. Código de esfuerzo, Gussenhoven, 2004). Por otro lado, en la figura 5 se puede observar la realización del acento nuclear $\mathrm{L}+{ }_{j} \mathrm{H}^{*}$, con un ascenso de 5 st, que continúa con el movimiento en la sílaba postónica, con el tono de juntura $\mathrm{H} \%$. Esta configuración nuclear es diferente al tonema $\mathrm{L}^{*} \mathrm{LH} \%$ registrado en la variedad de la Ciudad de México (De la Mota et al., 2010).

Respecto a la configuración de la pregunta eco en esta variedad del español, se registran dos tonemas: i) con el acento nuclear $\mathrm{L}+\mathrm{H}^{*}$, con la variante $\mathrm{L}+{ }_{j} \mathrm{H}^{*}$ - ascenso superior a 4 semitonos- y ii) con el acento nuclear monotonal bajo $L^{*}$, similar al registrado en la variedad de la Ciudad de México (De la Mota 
et al., 2010). En ambos casos el tonema incluye un tono de juntura ${ }_{i} \mathrm{H} \%$, que indica un ascenso prominente, en un rango de 5 a 8 semitonos.

Para la elicitación de la pregunta eco se presentó el siguiente contexto:

(3) Te dicen la hora, pero no oíste bien. Crees que te dijeron que son las nueve. Pregunta si te dijeron que son las nueve (Respuesta esperada: ¿Dijiste que son las nueve? ¿Me dijiste que son las nueve?)

En los casos donde únicamente se repite la información que se busca confirmar, como en ¿Son las nueve? ¿ ¿Dijo las nueve?, el tonema se realiza con el acento nuclear monotonal $\mathrm{L}^{\star}{ }_{i} \mathrm{H} \%$. En contraste, cuando el enunciado se produce con una oración subordinada de objeto directo, introducida mediante la conjunción "que", como en ¿Me dijiste/Me dijeron que son las nueve?, el acento nuclear se realiza con la forma bitonal $\mathrm{L}+\left({ }_{\mathrm{i}}\right) \mathrm{H}^{*}{ }_{\mathrm{i}} \mathrm{H} \%$, como se muestra en el enunciado de la figura 6.

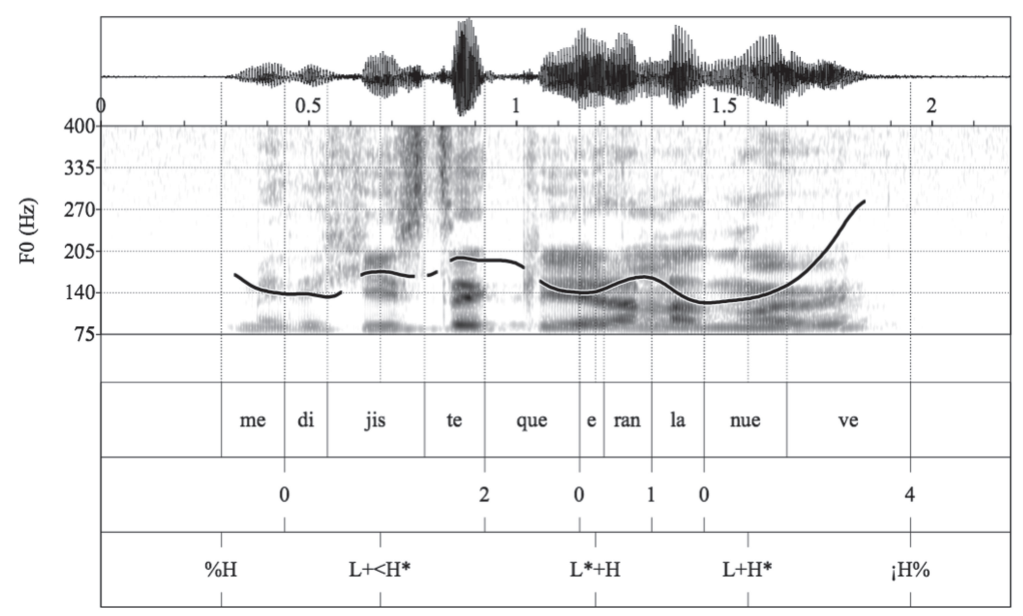

Figura 6. Oscilograma, espectrograma y curva melódica del enunciado ¿Me dijiste que eran la nueve? (sic), producido por un hombre, joven

El pretonema del enunciado de la figura 6 muestra una secuencia de acentos bitonales con desplazamiento en la sílaba 
postónica, seguidos del acento nuclear $\mathrm{L}+\mathrm{H}^{*}$ - con un ascenso de 2.5 semitonos - y el tono de juntura ${ }_{i} \mathrm{H} \%$, que se realiza con un movimiento de 6 semitonos. En este enunciado se puede apreciar un descenso que ocurre en la sílaba prenuclear /la-/, de manera que el tramo del tono bajo del acento nuclear $\mathrm{L}+\mathrm{H}^{*}$ inicia en el nivel del piso tonal del enunciado, el más bajo en comparación con los acentos bitonales del material prenuclear. Este descenso aporta una similitud con los acentos nucleares $L^{*}$ de las variedades de la Ciudad de México y Guadalajara.

Interrogativas absolutas imperativas (órdenes)

Las preguntas absolutas imperativas que cumplen una función de orden se producen con dos tonemas: i) con el acento nuclear bitonal $\mathrm{L}+{ }_{\mathrm{i}} \mathrm{H}^{*}$, y ii) con el acento nuclear monotonal (!) $\mathrm{H}^{*}$; en ambos casos con un tono de juntura alto $\mathrm{H} \%$, que se realiza con un movimiento ascendente menor a 5 semitonos. Además se distingue el tono de juntura inicial $\% \mathrm{H}$ en todos los casos. No se registran tonemas con el acento nuclear bajo $L^{*}$, y esto es una diferencia respecto a las interrogativas absolutas neutras y de confirmación. La figura 7 muestra un ejemplo del tonema $! \mathrm{H}^{\star} \mathrm{H} \%$.

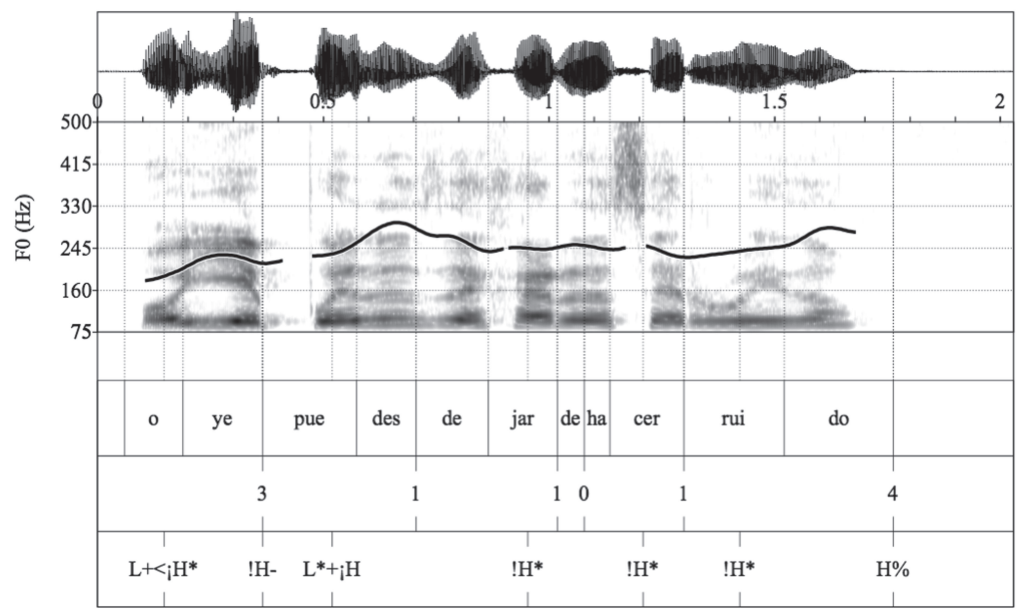

Figura 7. Oscilograma, espectrograma y curva melódica del enunciado

Oye, ¿puedes dejar de hacer ruido?, producido por una mujer, adulta 
El enunciado interrogativo de la figura 7 muestra el desplazamiento del primer acento tonal, en la palabra puedes - con un ascenso de 4.4 semitonos-, y a partir del pico tonal se produce un descenso de 3.6 semitonos hasta el segundo acento tonal $! \mathrm{H}^{*}$. Aunque en la curva melódica se aprecie un ascenso en la sílaba nuclear, éste se produce con un movimiento de 1 semitono, y el F0 se mantiene en un rango muy cercano al acento tonal anterior, con una diferencia entre 0.21 entre estos dos puntos. ${ }^{20}$ Finalmente, el tono de juntura $\mathrm{H} \%$ se realiza con un ascenso de 2.7 semitonos. Además, se puede notar que entre el primer pico tonal del enunciado, en la palabra puedes y la juntura final $\mathrm{H} \%$ hay una diferencia de 0.06 semitonos; de tal manera que se encuentran prácticamente en el mismo rango tonal.

En relación con el acento nuclear, se observó una diferencia en los datos de los hablantes mayores, respecto a los enunciados de los jóvenes y los adultos. En los enunciados del grupo de edad mayor, se registró el acento nuclear bitonal $\mathrm{L}+{ }_{\mathrm{j}} \mathrm{H}^{*}$ con ascenso superior a 3 semitonos — con 3.8 st para la mujer y 4.6 st para el hombre-; mientras que en los datos de los adultos y jóvenes se observa la realización del acento nuclear monotonal $! \mathrm{H}^{*}$. Ambos acentos nucleares son diferentes de la configuración $\mathrm{L}^{\star} \mathrm{LH} \%$ registrada en la variedad de la Ciudad de México (De la Mota et al., 2010).

La realización del tono de juntura final aporta una diferencia entre distintos tipos de preguntas. El promedio de ascenso en el tono de juntura $\mathrm{H} \%$ es de 2.8 semitonos, que se puede considerar moderado si se compara con los enunciados interrogativos absolutos neutros o confirmativos - incluyendo a los eco- en la misma variedad. A diferencia de los enunciados de la Ciudad de México - que registra el acento nuclear bajo $\mathrm{L}^{*}$ - , la realización de acentos nucleares con tendencia no descendente $\left(\mathrm{L}+{ }_{j} \mathrm{H}^{*}\right.$ $\left.\mathrm{y} ! \mathrm{H}^{\star}\right)$ podría explicar que el movimiento en la juntura sea poco elevado en la variedad de Morelia; de tal manera que el ascenso

\footnotetext{
${ }^{20}$ El parámetro de intensidad, con un valor similar en la sílaba tónica prenuclear "cer" de la palabra hacer y la nuclear en ruido (en un rango de $83 \mathrm{db}$ ) es una pista que apoya el anclaje de acentos tonales en sílabas prominentes en ambas palabras, a pesar del choque tonal que se produce.
} 
comienza desde el acento nuclear y no ocurre únicamente en la juntura.

\section{Interrogativas absolutas exhortativas. Invitaciones}

En las preguntas absolutas que cumplen una función de invitación, se registra el tonema (!) $\mathrm{H}^{*}{ }_{i} \mathrm{H} \%$, con un acento nuclear monotonal alto, seguido de un marcado ascenso en la juntura final - en un rango de 3 a 6 semitonos-. La forma monotonal del acento nuclear representa la realización de movimientos menores a 1 semitono en la sílaba nuclear — que de hecho se encuentran en un rango de 0.5 semitonos-. El movimiento más prominente ocurre en la juntura final, como se observa en la figura 8.

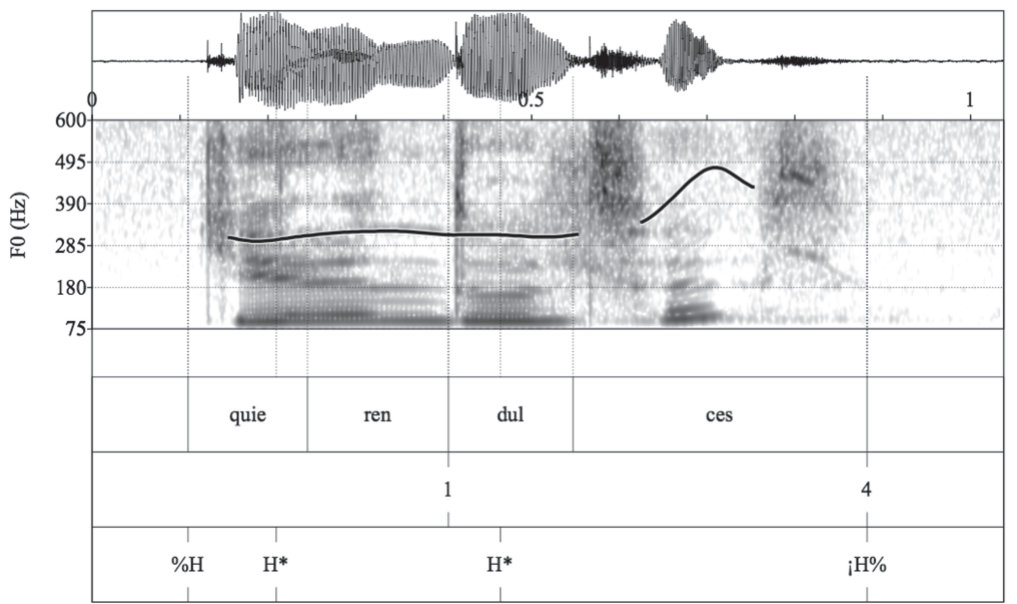

Figura 8. Oscilograma, espectrograma y curva melódica del enunciado ¿Quieren dulces?, producido por una mujer, joven

La figura 8 muestra la realización de un acento tonal alto $\mathrm{H}^{*}$ en el pretonema y el tonema, seguido de un ascenso de 6 st en la juntura final $¡ \mathrm{H} \%$. El enunciado se realiza en un rango alto desde el primer acento tonal y la curva melódica permanece sin inflexiones hasta la juntura del enunciado. El nivel alto del 
enunciado se observa desde el tono de juntura inicial \%H, que se registró de manera casi categórica en todos los enunciados de este tipo, excepto en los datos de la mujer mayor. En el pretonema hay un predominio de acentos tonales con alineamiento en la sílaba tónica - ya sea el acento monotonal $\mathrm{H}^{*}$ o bitonal $\mathrm{L}+\mathrm{H}^{\star}$-, excepto en los datos de la mujer del grupo de adultos, que registra el acento bitonal $\mathrm{L}^{*}+\mathrm{H}$, con desplazamiento del pico tonal. El alineamiento al interior de la sílaba tónica en el primer acento tonal contrasta con el desplazamiento del pico tonal, registrado en los enunciados interrogativos absolutos neutros y de las órdenes (cf. figura 7).

La configuración nuclear y prenuclear de las interrogativas con función de invitación en los datos de Morelia es idéntica a la registrada en Guadalajara (cf. Orozco, 2017: 296), incluso en el valor del ascenso de 6 st en el tono de juntura final. Estas variedades difieren de la configuración registrada en los datos de la Ciudad de México, con el acento prenuclear alineado en la sílaba postónica $\mathrm{L}^{*}+\mathrm{H}$ y el acento nuclear bajo $\mathrm{L}^{*}$ (De la Mota et al., 2010). Sin embargo, en las tres variedades se realiza un ascenso prominente en la juntura final.

\section{Configuraciones tonales de enunciados interrogativos pronominales $(q u-)$}

\section{Interrogativa qu- neutra (búsqueda de información)}

Para la variedad del español de Morelia se observa una mayor variación en los tonemas de las interrogativas $q u$ - neutras, en comparación con las preguntas absolutas neutras. Se registran cinco tonemas: i) $\mathrm{L}^{*} \mathrm{~L} \%$ y ii) $\mathrm{H}+\mathrm{L}^{*} \mathrm{~L} \%$, con patrón descendente; iii) $\mathrm{L}^{\star} \mathrm{H} \%$ y iv) $\mathrm{L}+\mathrm{H}^{*} \mathrm{H} \%$, con junturas ascendentes; y v) $\mathrm{H}^{\star}$ $\mathrm{H} \%$ / !H\%, con ascenso o sostenimiento del tono al final. A diferencia de la variedad de la Ciudad de México, en los datos de Morelia no se registran casos de acento nuclear bitonal con juntura descendente $\mathrm{L}+\mathrm{H}^{\star} \mathrm{HL} \%$, sino que el movimiento de ascenso del acento nuclear $\mathrm{L}+\mathrm{H}^{\star}$ se prolonga hasta la sílaba postónica 
(véase figura 10 infra). Otra particularidad de la interrogativa neutra $q u$ - es la recurrencia del tono de juntura inicial \%H.

En posición nuclear, las formas bitonales se registraron principalmente en los datos de los hablantes mayores (hombre y mujer), en contraste con las realizaciones de los jóvenes y adultos que registran mayor ocurrencia de acentos nucleares $\mathrm{L}^{*} \mathrm{e}$ incluso con descenso $\mathrm{H}+\mathrm{L}^{\star}$, además del acento nuclear monotonal alto $\mathrm{H}^{*}$. La diferencia en la curva melódica de la configuración nuclear $\mathrm{L}^{*} \mathrm{~L} \%$ y $\mathrm{L}+\mathrm{H}^{*} \mathrm{H} \%$ se muestra en los enunciados de las figuras 9 y 10.

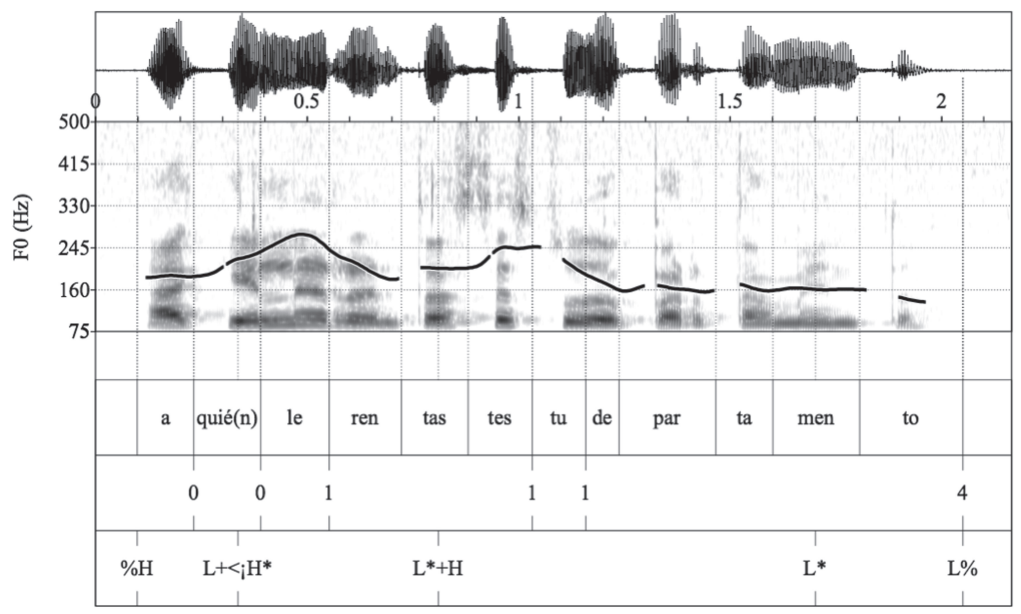

Figura 9. Oscilograma, espectrograma y curva melódica del enunciado ¿A quién le rentaste tu departamento?, producido por una mujer, adulta

El enunciado de la figura 9 muestra en la parte prenuclear una secuencia de acentos tonales con alineamiento en la sílaba postónica, de manera similar a los enunciados interrogativos absolutos. A partir de la culminación del segundo acento prenuclear se observa un marcado movimiento descendente - de 8 st - hasta el tonema del enunciado. ${ }^{21}$ Nótese que este descenso es mayor al que se produce en el enunciado interrogativo ab-

\footnotetext{
${ }^{21}$ Respecto a la configuración global, se registra una diferencia de 5 st entre el inicio absoluto y el tono de juntura L\% del enunciado.
} 
soluto, en el que se registra un movimiento descendente de 6 st para los datos de la misma hablante (cf. figura 4 supra).

Otra configuración nuclear se produce con un movimiento ascendente, con el acento nuclear bitonal $\mathrm{L}+\mathrm{H}^{\star}$ - con ascenso menor a 3 st - seguido de un tono de juntura final (!)H\%, que puede realizarse como el sostenimiento del tono alto del acento nuclear $\mathrm{L}+\mathrm{H}^{\star}$ o como un ascenso menor a 3 st. Sólo se registró un caso con ascenso de 5.7 st, en los datos de la mujer mayor (figura 10).

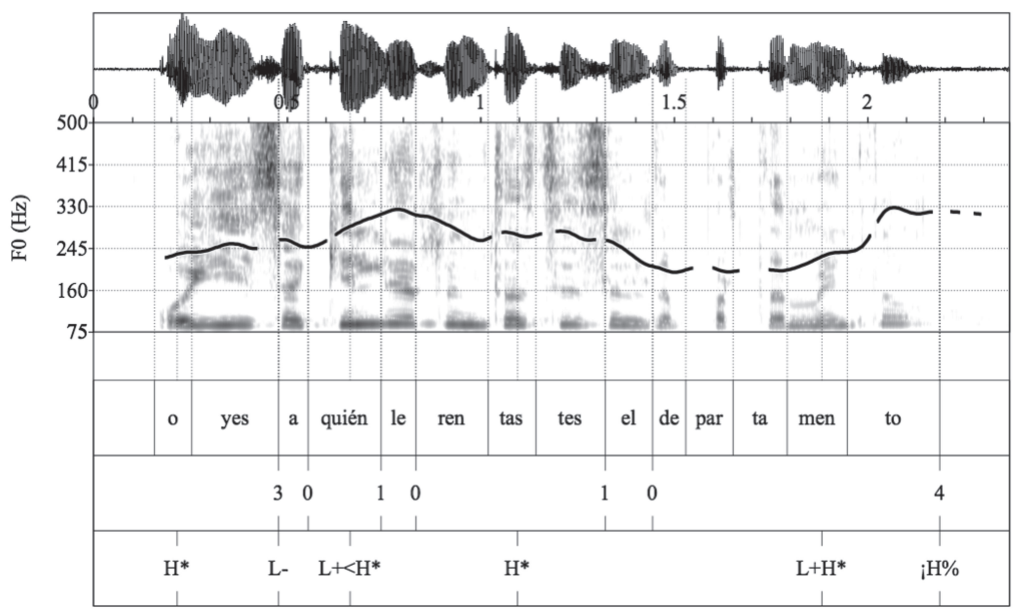

Figura 10. Oscilograma, espectrograma y curva melódica del enunciado Oyes, ¿a quién le rentaste el departamento?, producido por una mujer, mayor

En este enunciado se observa un descenso de 5 st desde el segundo acento prenuclear, en la palabra rentaste(s), hasta el inicio de la sílaba nuclear. La diferencia respecto al enunciado de la figura 9 ocurre en el acento nuclear bitonal $\mathrm{L}+\mathrm{H}^{*}$ que prolonga el ascenso hasta la sílaba postónica. También se puede notar que el nivel del tono de juntura coincide con el del primer pico tonal, asociado con la palabra qu- (ambos en un rango de $330 \mathrm{hz}$ ). El ascenso prominente de ${ }_{\mathrm{i}} \mathrm{H} \%$ puede asociarse con una mayor expresividad del hablante o con una mayor implicación en el acto de habla (cf. NGLE, 2011: 459 para el español en general; Estebas Vilaplana y Prieto, 2010: 35, para el español castellano). 
Interrogativa qu- de confirmación

Los ascensos prominentes en el tono de juntura también se registran en las preguntas $q u$ - de confirmación, que involucran una mayor expresividad del hablante. Se registró la configuración nuclear $\mathrm{L}+\mathrm{H}^{*}{ }_{i} \mathrm{H} \%$, con el acento nuclear bitonal, seguido de un tono de juntura alto, con ascenso superior a 5 st. La figura 11 muestra la realización de esta configuración, con un valor del ascenso de 9 st en la juntura ${ }_{i} \mathrm{H} \%$.

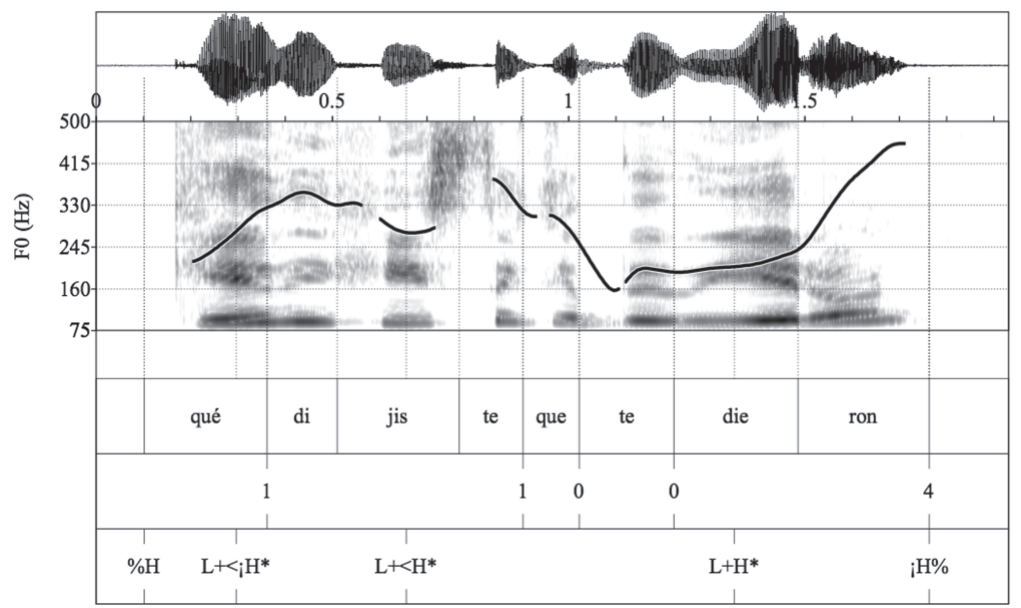

Figura 11. Oscilograma, espectrograma y curva melódica del enunciado ¿Qué dijiste que te dieron?, producido por una mujer, adulta

En este enunciado se puede notar que el pico tonal del tono de juntura ${ }_{i} \mathrm{H} \%$ es el punto más alto del enunciado, incluso más alto que el primer pico tonal que se asocia con la palabra interrogativa $q u-$, con una diferencia de 1.5 st entre ambos puntos. Respecto al acento nuclear, el movimiento en la sílaba tónica es de 2 st; sin embargo, el movimiento no se produce como un ascenso progresivo a lo largo de la sílaba tónica, sino que el ascenso ocurre en el inicio de la vocal /e-/, en la sílaba /die-/. En esta primera aproximación a los datos se tomó en cuenta, además del valor del movimiento, que el ascenso ocurre en el núcleo del diptongo, por lo tanto se asignó un acento bitonal $\mathrm{L}+\mathrm{H}^{*}$. La com- 
paración con otros tipos enunciativos - como el aseverativoy con otros estilos de habla, además de una prueba de percepción podrá apoyar la notación de este acento tonal como un bitono o si se trata de un ascenso preparatorio.

En esta muestra de datos se ha observado que el ascenso prominente - en un rango de 7 a 8 st- es una característica de las preguntas qu-de confirmación. Este rasgo se comparte con las interrogativas absolutas de confirmación en la misma variedad del español. Dicho ascenso se distingue del registrado en las interrogativas absolutas neutras, con un promedio de 3.5 st -y un valor máximo de 5 st- para el tono de juntura $\mathrm{H} \%$. En este sentido, podríamos sugerir que el ascenso prominente en la juntura final indica el carácter no neutro del enunciado interrogativo, pues no hay un desconocimiento completo de la respuesta, y además se agrega un matiz expresivo al enunciado.

\section{Interrogativa qu- imperativa (órdenes)}

En la pregunta imperativa $q u$ - con función de orden se observa una mayor variación en los tonemas, en comparación con las interrogativas absolutas que se formulan con el mismo propósito. Se registran tres realizaciones: i) $\mathrm{L}+(\mathrm{i}) \mathrm{H}^{*}{ }_{i} \mathrm{H} \%$, con ascenso en el acento nuclear y sostenimiento del tono de juntura final; ii) ${ }_{i} \mathrm{H}^{*} / \mathrm{L}^{*}($ !) $\mathrm{H} \%$, con sostenimiento del tono o una inflexión final, menor a 1.5 st; y iii) $\mathrm{L}^{\star} \mathrm{L} \%$, con terminación descendente. En este último caso, la configuración descendente aporta un matiz de mayor autoridad en la enunciación (cf. Código de frecuencia, Gussenhoven, 2004). La palabra interrogativa $q u$ - se produce con mayor prominencia en el enunciado, y tiene dos realizaciones: i) la forma bitonal $\mathrm{L}+<\mathrm{H}^{*}$, con pico tonal desplazado a la sílaba postónica; y ii) con el acento bitonal $\mathrm{L}+\mathrm{H}^{*}$, alineado al interior de la sílaba tónica. Un ejemplo de la realización con pico tonal desplazado se observa en la figura 12. 


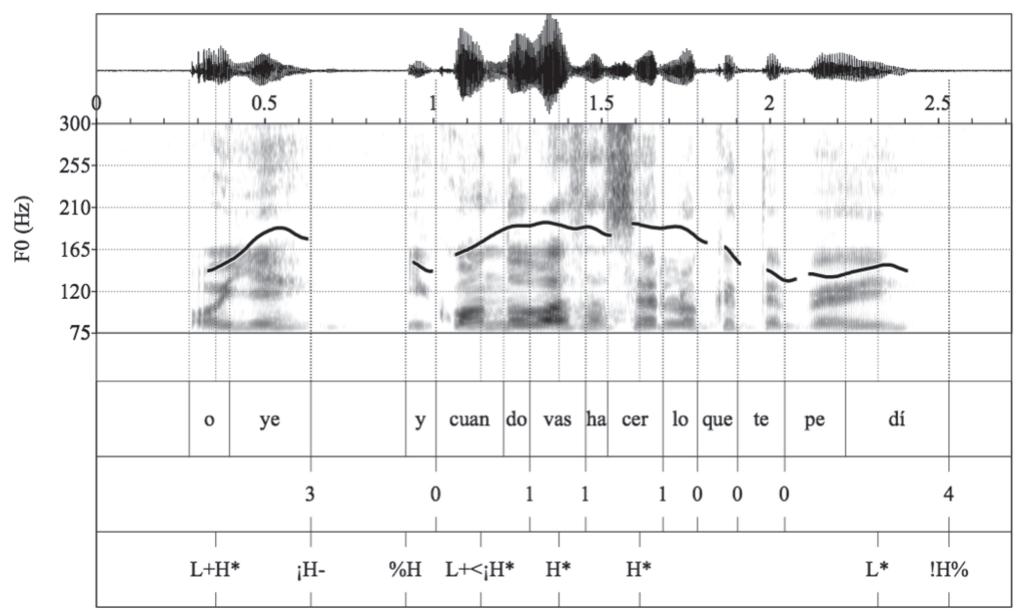

Figura 12. Oscilograma, espectrograma y curva melódica del enunciado Oye, ¿cuándo vas a hacer lo que te pedí?, producido por un hombre, joven

El enunciado de la figura 12 muestra en la palabra interrogativa $q u$ - el acento tonal $\mathrm{L}+<{ }_{i} \mathrm{H}^{*}$, seguido de una secuencia de acentos tonales altos $\mathrm{H}^{*}$. El descenso del enunciado comienza en sílaba posterior al pico tonal de la palabra hacer, y el valor del movimiento desde este punto hasta la sílaba prenuclear es de 5 st. En el tonema se observa una sutil inflexión ascendente en la sílaba nuclear - en la palabra pedí- pero el valor del ascenso es de 1 st, por lo tanto se considera como un acento monotonal, seguido de la juntura $! \mathrm{H} \%{ }^{22} \mathrm{El}$ tonema del enunciado con el acento nuclear bajo $\mathrm{L}^{*}$ es una diferencia respecto a las preguntas absolutas imperativas que cumplen una función de orden, donde éste no se registra.

Al establecer la diferencia con otras variedades del español de México, se observa que la configuración global es similar a la que se reporta en datos de la Ciudad de México para este tipo de interrogativas, con el pico tonal más alto en la palabra interrogativa qu- (De la Mota et al., 2010). La diferencia ocurre en el descenso del enunciado, que en la variedad del centro, se realiza de manera progresiva - con escalonamiento descendente, a partir

\footnotetext{
${ }^{22}$ En casos de mayor expresividad en el enunciado, se registra un movimiento de 4 st en el acento nuclear.
} 
del primer pico tonal-; en contraste con la variedad de Morelia, donde se observa una secuencia de tonos altos $\mathrm{H}^{\star}$ en el cuerpo del enunciado, y un abrupto descenso que ocurre a partir del último acento prenuclear.

\section{Interrogativa qu- exhortativas. Invitaciones ${ }^{23}$}

En las invitaciones con forma de pregunta qu-se observó menor variación en la realización de los tonemas. Se registraron dos configuraciones nucleares: i) $\mathrm{L}^{*} \mathrm{~L} \%$ con terminación descendente —en datos de jóvenes y adultos-; y ii) $\mathrm{L}+\mathrm{H}^{\star} ! \mathrm{H} \%$, con ascenso y/o sostenimiento del tono de juntura final —en datos de hablantes mayores (véase figura 13) - En los casos de ascenso, el movimiento es de 1.5 a 3 st, y este puede ocurrir en el acento nuclear o en la juntura final. De tal manera que los ascensos en estos enunciados son menores que los registrados en las órdenes con forma de pregunta qu-.

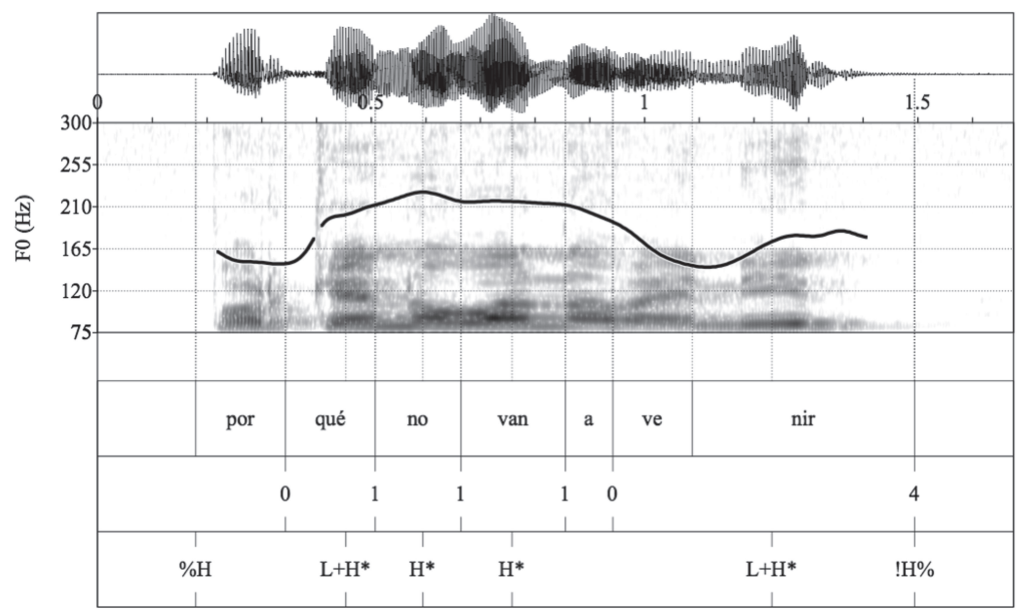

Figura 13. Oscilograma, espectrograma y curva melódica del enunciado ¿Por qué no van a venir?, producido por un hombre, mayor

\footnotetext{
${ }^{23}$ Este término se retoma de la clasificación de la NGLE (2011: 471). Se consideran enunciados no neutros porque el hablante no busca obtener información que desconoce, sino hacer un ofrecimiento y tratar de convencer al interlocutor para que realice una acción.
} 
El pretonema se realiza con el alineamiento del pico tonal al interior de la sílaba tónica, es decir con los acentos tonales $\mathrm{L}+\mathrm{H}^{\star} \mathrm{y} \mathrm{H}^{\star},{ }^{24}$ como se muestra en el enunciado de la figura 13. Este alineamiento es similar al que se observa en las invitaciones con pregunta absoluta. El tonema del enunciado se realiza con el acento nuclear $\mathrm{L}+\mathrm{H}^{*}$, con un ascenso de 3 semitonos, seguido del sostenimiento del tono alto en la juntura final. Respecto a la configuración global, a diferencia de las órdenes con interrogativa $q u$-, en este caso el pico tonal más alto ocurre en los dos primeros acentos prenucleares, y no en la juntura (cf. figura 11 supra). Además se observa un marcado descenso de 7 st que se produce desde el último acento prenuclear —en la palabra van- hacia la sílaba nuclear, con una excursión tonal más amplia en el contexto exhortativo.

El alineamiento en la sílaba tónica de los acentos prenucleares $\left(\mathrm{L}+\mathrm{H}^{*}, \mathrm{H}^{*}\right)$, y en el tonema $\mathrm{L}+{ }_{i} \mathrm{H}^{*} ! \mathrm{H} \%$ es similar al que se registra en datos de la Ciudad de México (cf. De la Mota et al., 2010). ${ }^{25}$ La diferencia entre las dos variedades se observa en la forma de la curva melódica, con el nivel del F0 más alto en el tonema del enunciado de la Ciudad de México; en contraste con la variedad de Morelia, que muestra este rasgo en el pretonema del enunciado. De tal manera que aunque ambas variedades registren la misma configuración nuclear y prenuclear, estas se distinguen por medio de particularidades fonéticas.

\footnotetext{
${ }^{24}$ Otra propuesta de transcripción es considerar que en la palabra interrogativa se produce un pico desplazado hacia la sílaba postónica. En este caso de choque tonal, ambas sílabas conservan su prominencia, por lo tanto se asigna un acento tonal para cada una de las palabras monosilábicas. El hecho de que ambas sílabas conserven su prominencia puede atribuirse al matiz de insistencia con el que se produce el enunciado. Agradezco la observación de uno de los dictaminadores acerca de la transcripción de esta secuencia de acentos tonales.

${ }^{25}$ En la notación prosódica propuesta por De la Mota et al. (2010), la juntura se indica con el tono medio $\mathrm{M} \%$, que equivale a $\mathrm{H} \%$, de acuerdo con la propuesta de notación que se utiliza en Hualde y Prieto (2015).
} 


\section{Conclusiones preliminares}

En este trabajo se han descrito las configuraciones tonales de distintos tipos de enunciados interrogativos en la variedad de Morelia, Michoacán. En las preguntas absolutas neutras se registra el tonema $\mathrm{L}+\mathrm{H}^{*} \mathrm{H} \%$, con un acento nuclear bitonal, que se distingue de las variedades de Guadalajara y Ciudad de México. La realización del acento nuclear, con un moderado movimiento en la sílaba tónica plantea la cuestión acerca de un posible ascenso preparatorio - que distinguiría a la variedad de Morelia.

En el caso de las preguntas $q u^{-}$, la realización del tonema $\mathrm{L}+\mathrm{H}^{*} \mathrm{H} \%$ en preguntas neutras o de confirmación se relaciona con una mayor expresividad o involucramiento del hablante en el acto de habla. Por otro lado, la variante $\mathrm{L}+{ }_{j} \mathrm{H}^{*} \mathrm{H} \%-$ con ascenso superior a 3 st en el acento nuclear- se realiza en preguntas absolutas de confirmación (expresivas), pregunta eco y órdenes; es decir, el ascenso prominente se asocia con interrogativos no neutros.

El tono de juntura final de las interrogativas absolutas - neutras y no neutras - aporta otra diferencia entre las tres variedades. En los datos de Morelia, el punto de ascenso del tono de juntura final ocurre en la parte inicial de la sílaba postónica $(\mathrm{H} \%)$; en contraste con la variedad del centro y de Guadalajara, donde el ascenso ocurre en la parte media de la sílaba postónica (LH\%). Por otro lado, el valor del movimiento en la juntura de los interrogativos absolutos neutros es otro parámetro que distingue a las variedades de Morelia y Guadalajara. En ambas variedades se reporta un ascenso de 3.5 st — con un rango máximo de 5 st en Morelia-; sin embargo, en la variedad de Guadalajara el ascenso puede alcanzar hasta 10 st (Orozco, 2017), lo que sugiere la producción de ascensos finales más prominentes en esta variedad. En los datos de Morelia, los ascensos superiores a $5 \mathrm{st}$ en el tono de juntura se registran en interrogativas no neutras, es decir, en aquellas en las que el objetivo del hablante no es la búsqueda de información - preguntas absolutas y qu- de confirmación e imperativas (órdenes). 
Por otro lado, se observó que el inicio de los enunciados interrogativos $q u$ - tienen un nivel más alto en comparación con los interrogativos absolutos y los aseverativos. Esta tendencia es similar a la reportada para otras variedades de hablas centrales. La pertinencia del tono de juntura inicial se podrá determinar con un análisis que incorpore umbrales de comparación entre tipos enunciativos - por ahora se ha observado la diferencia de 1 a 1.5 st entre el tono normal y los interrogativos.

Las configuraciones registradas en este trabajo requieren de un estudio más detallado que incorpore distintas variables —sociales, pragmáticas, etc.- y su comparación con otros tipos enunciativos. La posible influencia de los factores sociales se observó en la realización de acentos nucleares monotonales en datos de los hablantes más jóvenes, en contraste con las formas bitonales, en datos de mayores. Esta tendencia se tendrá que comprobar en una muestra de datos que incluya diferentes estilos de habla y un mayor número de hablantes.

Con esta aproximación a los datos, se pueden formular hipótesis acerca de las configuraciones tonales que se producirían para un determinado tipo enunciativo en un contexto natural. En todo caso, es imprescindible que en investigaciones posteriores se analicen datos elicitados en contextos espontáneos e incorporar factores sociolingüísticos que puedan dar cuenta de la variación de los patrones entonativos.

\section{Bibliografía}

Ávila, S. (2003), "La entonación del enunciado interrogativo en el español de la ciudad de México", en E. Herrera Zendejas y P. Martín Butragueño (eds.), La tonía: Dimensiones fonéticas y fonológicas, México, El Colegio de México, pp. 331-355.

Armstrong, M. E. (2010), "Puerto Rican Spanish intonation”, en P. Prieto y P. Roseano (eds.), Transcription of intonation of the Spanish Language, Múnich, Lincom Europa, pp. 155190. 
Beckman M., M. Díaz-Campos, J. T Mcgory y T. A. Morgan (2002), "Intonation across Spanish, in the tones and break indices framework", Probus, 14, pp. 9-36.

Boersma, P. y D. Weenink (2016), Praat. Versión 6.0.16, Ámsterdam, Universidad de Ámsterdam.

Coronado Hernández, J. (2014), “Entonación rural del español de México”, en P. Martin Butragueño y L. Orozco (eds.), Argumentos cuantitativos y cualitativos en sociolingüística, México, El Colegio de México, pp. 61-77.

Dorta, J., J. A. Martín y C. Jorge (2018), "La entonación de Canarias", en J. Dorta (ed.), La entonación declarativa e interrogativa en cinco zonas fronterizas del español, Fráncfort, Peter Lang, pp. 85-107.

EsCANDELL, V. (1993), Introducción a la pragmática, Barcelona / Madrid, Anthropos / Universidad Nacional de Educación a Distancia.

Estebas Vilaplana, E. y P. Prieto (2008), "La notación prosódica del español: una revisión del Sp_ToBI”, Estudios de Fonética Experimental, 17, pp. 263-283.

Estebas Vilaplana, E. y P. Prieto (2010), “Castilian Spanish Intonation”, en P. Prieto y P. Roseano (eds.), Transcription of intonation of the Spanish Language, Múnich, Lincom Europa, pp. 17-48.

FACE, T. (2004), "Intonation of absolute interrogatives in Castilian Spanish”, Southwest Journal of Linguistics, 23:2, pp. 65-79. Gussenhoven, C. (2004), The Phonology of Tone and Intonation, Cambridge, Cambridge University Press.

Hualde, J. I. y P. Prieto (2015), "Intonational variation in Spanish: European and American varieties", en S. Frota y P. Prieto (eds.), Intonation in Romance, Oxford, Oxford University Press, pp. 350-391.

INEGI (2015), México en cifras. Morelia, Michoacán de Ocampo. Instituto Nacional de Estadística y Geografía, en <https://www.inegi.org.mx/app/areasgeograficas/ ?ag $=16 \#>$ [consultado el 28 de noviembre de 2018].

LADD, R. (2008), Intonational Phonology, Cambridge, Cambridge University Press. 
Lope Blanch, J. M. (1971), "El léxico de la zona maya en el marco de la dialectología mexicana”, Nueva Revista de Filología Hispánica, 20:1, pp. 1-63.

Lope Blanch, J. M. (1979), Investigaciones sobre dialectología mexicana, México, Universidad Nacional Autónoma de México.

Lope Blanch, J. M. (1996), "México”, en M. Alvar (dir.), Manual de dialectología hispánica. El español de América, Barcelona, Ariel, pp. 81-89.

Martín, J. A. y J. Dorta (2018), "La entonación de Cuba”, en J. Dorta (ed.), La entonación declarativa e interrogativa en cinco zonas fronterizas del español, Fráncfort, Peter Lang, pp. 109-129.

Martín Butragueño, P. (2011), "La estratificación sociolingüística de la entonación circunfleja mexicana”, en P. Martín Butragueño (ed.), Realismo en el análisis de corpus orales. Primer coloquio de cambio y variación lingüística, México, El Colegio de México, pp. 93-121.

Martín Butragueño, P. (2014a), "Prosodia fonética de enunciados representativos e interrogativos absolutos: elementos locales y globales", Estudios de Fonética Experimental, 23, pp. 125-202.

Martín Butragueño, P. (2014b), Fonología variable del español de México. Volumen 1: procesos segmentales, México, El Colegio de México.

Martín Butragueño, P. (2016), “A veces lloro mis lágrimas. Acercamiento multivariable a la prosodia de los actos de habla expresivos en el español de México", Estudios de Lingüística Aplicada, 34:63, pp. 59-102.

Martín Butragueño, P., É. Mendoza y L. Orozco (coords.) (en preparación), Corpus oral del español de México (COEM), México, El Colegio de México.

Martínez Celdrán, E. y A. M. Fernández Planas (coords.) (2003-2018), Atlas Multimedia de la Prosodia del Espacio Románico <http://stel.ub.edu/labfon/amper/cast/index_ ampercat.html $>$ [consultado el 31 de enero de 2018].

Mendoza VÁzquez, É. (2014), La impresión de un tono: estudio 
sociolingüístico de la entonación de Cuapiaxtla, Tlaxcala, México, El Colegio de México, tesis de doctorado en Lingüística.

Montellano Moreno, U. (2017), Entonación de enunciados declarativos e interrogativos en el habla de Monterrey, México, Escuela Nacional de Antropología e Historia, tesis de licenciatura en Lingüística.

Mota, C. De la, P. Martín Butragueño y P. Prieto (2010), "Mexican Spanish Intonation", en P. Prieto y P. Roseano (eds.), Transcription of intonation of the Spanish Language, Múnich, Lincom Europa, pp. 319-350.

Murrieta Bello, L. (2016), Análisis experimental del umbral de percepción entonativa en el español del centro de México, México, Universidad Nacional Autónoma de México, tesis de licenciatura en Lengua y Literatura Hispánicas.

Navarro Tomás, T. (1944), Manual de entonación española, Nueva York, Hispanic Institute in the United States.

Nespor, M. e I. Vogel (1994), La prosodia, Madrid, Visor.

Noотевоом, S. (1997), "Prosody of speech: Melody and rhythm", en W. J. Hardcastle y J. Laver (eds.), The Handbook of Phonetic Sciences, Oxford, Blackwell, pp. 640-673.

Orozco, L. (2008), "Peticiones corteses y factores prosódicos", en E. Herrera y P. Martín Butragueño (eds.), Fonología instrumental: Patrones fónicos y variación, México, El Colegio de México, pp. 335-355.

Orozco, L. (2012), "Propuesta de clasificación de las situaciones del ATLES en tipos de actos de habla", Presentación en el Seminario de Prosodia, México, El Colegio de México, 12 de junio.

Orozco, L. (2017), "Variación dialectal en interrogativas absolutas. Datos de Guadalajara, Monterrey y Tuxtla Gutiérrez", en L. Orozco y A. Guerrero Galván (coords.), Estudios de variación geolingüística, México, Secretaría de Cultura / Instituto Nacional de Antropología e Historia, pp. 281-302. Ortiz, H., M. Fuentes y L. Astruc (2010), “Chilean Spanish intonation”, en P. Prieto y P. Roseano (eds.), Transcription 
of intonation of the Spanish Language, Múnich, Lincom Europa, pp. 255-284.

Pamies Bertrán, A., A. M. Fernández Planas, E. Martínez Celdrán, A. Ortega Escandell y M. Amorós CéspeDES (2001), "Umbrales tonales en español peninsular", en M. Cuenca Villain (ed.), Actas del II Congreso Nacional de Fonética Experimental, Sevilla, Universidad de Sevilla, pp. 272-278.

Pierrehumbert, J. (1980), The Phonetics and phonology of English Intonation, Massachusetts Institute of Technology, tesis de doctorado.

Prieto, P. (2004), “The search for phonological targets in the tonal space: $\mathrm{H} 1$ scaling and alignment in five sentencetypes in Peninsular Spanish", en T. L. Face (ed.), Laboratory approaches to Spanish phonology, Berlín, Mouton de Gruyter, pp. 29-59.

Prieto, P. y P. Roseano (coords.) (2009-2013), Atlas interactivo de la entonación del español <http://prosodia.upf.edu/atlasentonacion/> [consultado el 31 de enero de 2018].

Prieto, P. y G. Rigau (2011), "Prosody and pragmatics", en Ll. Payrató y J. M. Cots (eds.), The Pragmatics of Catalan, La Haya, Mouton de Gruyter, pp. 17-48.

Quilis, A. (1993), Tratado de fonología y fonética españolas, Madrid, Gredos.

Real Academia Española (2011), Nueva gramática de la lengua española. Fonética y Fonología, Madrid, Espasa-Calpe.

Sosa, J. M. (1999), La entonación del español. Su estructura fónica, variabilidad y dialectología, Madrid, Cátedra.

SosA, J. M. (2003), "Wh- questions in Spanish: Meanings and configuration variability", Catalan Journal of Linguistics, 2, Special Issue on Romance Intonation, vol. 2, Barcelona, Universidad Autónoma de Barcelona, pp. 229-247.

WiLlis, E. W. (2008), “Tonal characteristics of pronominal interrogatives in Puebla Mexico Spanish", en E. Herrera y P. Martín Butragueño (eds.), Fonología instrumental: Patrones fónicos y variación, México, El Colegio de México, pp. 357-375. 

Zamora Munné, J. C. y J. M. Guitart (1982), Dialectología hispanoamericana. Teoría-Descripción- Historia, Salaman- ca, Almar.

Welby, P. (2003), CreaFigures Script.praat [modificado en 2009 por Rafèu Sichel-Bazin].

Érika Mendoza Vázquez es investigadora Asociado C de tiempo completo en el Centro de Lingüística Hispánica "Juan M. Lope Blanch", en el Instituto de Investigaciones Filológicas UNAM. Áreas de Investigación: Sociofonética, Variación lingüística, Entonación y Prosodia

Licenciada en Lingüística por la Universidad Autónoma Metropolitana-Iztapalapa (2008) y Doctora en Lingüística por El Colegio de México (2014). Los principales intereses de su investigación son la prosodia y entonación en distintas variedades del español de México en entornos urbanos y rurales. Ha trabajado las propiedades prosódicas del foco contrastivo e informativo en la Ciudad de México.

Su actividad docente se ha llevado a cabo en la Maestría de Lingüística Hispánica de la UNAM y en la Escuela Nacional de Antropología e Historia. También ha participado en talleres de análisis instrumental en el Instituto Nacional de Lenguas Indígenas (INALI).

Actualmente participa en el Seminario de Prosodia (COLMEX-UNAM-UAQ) y es co-coordinadora del Corpus Oral del Español de México (COEM, Martín Butragueño, Mendoza y Orozco en preparación). Actualmente desarrolla el proyecto de investigación "Configuraciones tonales del español de México. Bases para una caracterización geoprosódica", enfocado en la descripción de las configuraciones tonales de distintas ciudades de México. La Paz, Chihuahua, Morelia y Tuxtla Gutiérrez. 